Draft Version November 19, 2018

Preprint typeset using $\mathrm{IAT}_{\mathrm{E}} \mathrm{X}$ style emulateapj v. 5/2/11

\title{
DISSIPATIONLESS FORMATION AND EVOLUTION OF THE MILKY WAY NUCLEAR STAR CLUSTER
}

\author{
FABio Antonini \\ Canadian Institute for Theoretical Astrophysics, University of Toronto, 60 St. George Street, Toronto, Ontario M5S 3H8, Canada
}

Roberto CApuzzo-Dolcetta

Department of Physics, Sapienza-Università di Roma, P.le A. Moro 5, I-00185, Rome, Italy

Alessandra Mastrobuono-Battisti

Department of Physics, Sapienza-Università di Roma, P.le A. Moro 5, I-00185, Rome, Italy

DAVID MERRITT

Department of Physics and Center for Computational Relativity and Gravitation, Rochester Institute of Technology, 85 Lomb Memorial Drive, Rochester, NY 14623, USA

Draft version November 19, 2018

\begin{abstract}
In one widely discussed model for the formation of nuclear star clusters (NSCs), massive globular clusters spiral into the center of a galaxy and merge to form the nucleus. It is now known that at least some NSCs coexist with supermassive black holes (SBHs); this is the case, for instance, in the Milky Way. In this paper, we investigate how the presence of a SMBH at the center of the Milky Way impacts the merger hypothesis for the formation of its NSC. Starting from a model consisting of a low-density nuclear stellar disk and the $\mathrm{SMBH}$, we use direct $N$-body simulations to follow the successive inspiral and merger of globular clusters. The clusters are started on circular orbits of radius $20 \mathrm{pc}$, and their initial masses and radii are set up in such a way as to be consistent with the galactic tidal field at that radius. These clusters, decayed orbitally in the central region due to their large mass, were followed in their inspiral events; as a result, the total accumulated mass by $\approx 10$ clusters is about $1.5 \times 10^{7} M_{\odot}$. Each cluster is disrupted by the SMBH at a distance of roughly one parsec. The density profile that results after the final inspiral event is characterized by a core of roughly this radius, and an envelope with density that falls off $\rho \sim r^{-2}$. These properties are similar to those of the Milky Way NSC, with the exception of the core size, which in the Milky Way is somewhat smaller. But by continuing the evolution of the model after the final inspiral event, we find that the core shrinks substantially via gravitational encounters in a time (when scaled to the Milky Way) of 10 Gyr as the stellar distribution evolves toward a Bahcall-Wolf cusp. We also show that the luminosity function of the Milky Way NSC is consistent with the hypothesis that 1/2 of the mass comes from old $(\sim 10 \mathrm{Gyr})$ stars, brought in by globular clusters, with the other half due to continuous star formation. We conclude that a model in which a large fraction of the mass of the Milky Way is due to infalling globular clusters is consistent with existing observational constraints.

Subject headings: galaxies: Milky Way Galaxy- Nuclear Clusters - stellar dynamics - methods: numerical, $N$-body simulations
\end{abstract}

\section{INTRODUCTION}

The centers of low-luminosity spheroids, $M_{B} \gtrsim-18$, are often marked by the presence of compact stellar nuclei with half-light radii of a few parsecs and luminosities that are $\sim 20$ times that of a typical globular cluster (Carollo et al. 1998; Böker et al. 2002; Côté et al. 2006). The nearest such system is at the center of our galaxy (Schödel et al. 2011). The Milky Way NSC is close enough that its radial and kinematical structure can be resolved (Schödel et al. 2007; Schödel et al. 2009). Its total mass is estimated at $\sim 10^{7} M_{\odot}$ (Launhardt et al. 2002; Schödel et al. 2008) and its half-light radius is roughly 3-5 pc (Graham \& Spitler 2009; Schödel et al. 2009, 2011). There is a central core of radius $\sim 0.5$ pc (Buchholz et al. 2009) , beyond which

antonini@cita.utoronto.ca the density falls off roughly as $r^{-1.8}$ (Genzel et al. 2003; Schödel et al. 2007; Oh et al. 2009).

NSCs with properties similar to those of the Milky Way have now been detected in galaxies of all Hubble types; a review of their properties is given by Böker (2010). NSCs exhibit complex star formation histories; while the bulk of the stars appear to always be old, the fraction of young stars increases toward late-type galaxies. In galaxies beyond the Local Group, NSCs are typically unresolved, and the only structural properties that can be derived are half-light radii and total luminosities. The study of NSCs has raised considerable interest because of the fairly strong correlations between their masses and the properties (mass, velocity dispersion) of their host galaxies (Ferrarese et al. 2006; Wehner \& Harris 2006). These correlations suggest that the formation of NSCs and their host galaxies are linked in important ways. 
Two models for the formation of NSCs have been widely discussed. In the in-situ formation model, buildup of molecular gas near the center of a galaxy leads to episodic star formation events (e.g., Loose et al. 1982). In this model, a NSC consists mostly of stars that formed locally (Schinnerer et al. 2006, 2008). A number of mechanisms have been discussed for bringing the gas to the center, including the magneto-rotational instability in a differentially rotating gas disk (Milosavljević 2004), tidal compression in shallow density profiles (Emsellem \& van de Ven 2008) or dynamical instabilities (Shlosman \& Begelman 1989; Bekki 2007).

Alternatively, in the merger model, globular clusters sink to the center of a galaxy via dynamical friction and merge to form a compact stellar system (Tremaine et al. 1975; Capuzzo-Dolcetta 1993; Agarwal \& Milosavljević 2011). Observations of NSCs in dwarf elliptical galaxies suggest that the majority of such nuclei might have formed in this way (Lotz et al. 2004). Numerical simulations have also shown that the basic properties of NSCs are consistent with a merger origin (Bekki et al. 2004; Capuzzo-Dolcetta \& Miocchi 2008; Hartmann et al. 2011).

In addition to a NSC, the Milky Way also contains a massive black hole $(\mathrm{SMBH})$ whose mass, $M_{\bullet} \approx 4 \times$ $10^{6} M_{\odot}$ (Ghez et al. 2008; Gillessen 2009), is comparable with that of the NSC. A handful of other galaxies are also known to contain both a NSC and a SMBH (Seth et al. 2008; Graham \& Spitler 2009), and the ratio of SMBH to NSC mass in these galaxies is of order unity. The possibility of a direct link between the population of intermediate mass black holes that might form in orbitally decaying star clusters and the growth of supermassive black holes at the center of galaxies has also been suggested in previous papers (e.g., Ebisuzaki et al. 2001; Portegies Zwart et al. 2006).

A simple argument leads to a $1 \mathrm{pc}$ scale as the relevant one for the merger model for the formation of NSCs. The beginning of the disruption process of a globular cluster due to tidal stresses from a SMBH is expected when it passes within a certain distance of the galaxy center, limiting the density within that radius. Disruption occurs at a distance $r=r_{\text {disr }}$ from the $\mathrm{SMBH}$, where

$$
\frac{M_{\bullet}}{\frac{4}{3} \pi r_{\text {disr }}^{3}} \approx \rho(0) \approx \frac{9}{4 \pi G} \frac{\sigma_{K}^{2}}{r_{K}^{2}} .
$$

Here $\rho(0)$ is the central (core) density of the globular cluster, $\sigma_{K}$ its central, one-dimensional velocity dispersion, and $r_{K}$ its core radius; the second relation is the "core-fitting formula" (King 1966). Writing

$$
r_{\mathrm{infl}} \equiv \frac{G M_{\bullet}}{\sigma_{\mathrm{NSC}}^{2}} \approx 1.3 \mathrm{pc}\left(\frac{M_{\bullet}}{4 \times 10^{6} M_{\odot}}\right)\left(\frac{\sigma_{\mathrm{NSC}}}{100 \mathrm{~km} \mathrm{~s}^{-1}}\right)^{-2}
$$

for the gravitational influence radius of the $\mathrm{SMBH}$, where $\sigma_{\mathrm{NSC}}$ is the stellar velocity dispersion in the NSC, equation (1) becomes

$$
r_{\mathrm{disr}} \approx 2\left(\frac{\sigma_{\mathrm{NSC}}}{5 \sigma_{K}}\right)^{2 / 3}\left(\frac{r_{\mathrm{inf}}}{r_{K}}\right)^{1 / 3} r_{K}
$$

Setting $r_{K}=0.5 \mathrm{pc}$ and $\sigma_{K}=20 \mathrm{~km} \mathrm{~s}^{-1}$, values characteristic of the most massive globular clusters, we find $r_{\text {disr }} \approx 1$ pc for the Milky Way. This is roughly equal to the radius of the core $(\sim 0.5 \mathrm{pc})$ that is observed in the distribution of late-type stars (Buchholz et al. 2009).

In this paper, we use direct $N$-body simulations to test the merger model for the formation of the Milky Way NSC. Our initial conditions consist of a SMBH and a diffuse stellar component that models the inner parts of the nuclear stellar disk (Launhardt et al. 2002). The NSC is built up by the successive inspiral of globular clusters, which we inject into the system at a radius of 20 pc. The clusters are assigned masses and radii consistent with those of globular clusters that were initially very massive $\left(\sim 4 \times 10^{6} M_{\odot}\right)$ but which were tidally limited by the Galaxy's tidal field. As the clusters spiral in due to dynamical friction against the stars in the disk component, they eventually come within the radius of tidal disruption of the SMBH. We follow 12 such inspirals, resulting in the accumulation of $\sim 10^{7} M_{\odot}$. The NSC that results has properties that are consistent with the observed properties of the Milky Way NSC, including a $\rho \sim r^{-2}$ density profile and a parsec-scale core.

At the Galactic center, the relaxation time at SgrA*'s influence radius is roughly $20-30 \mathrm{Gyr}$, assuming Solarmass stars (Merritt 2010). This is too long for a BahcallWolf (1976) cusp to have formed over the Galaxy's lifetime, consistent with the observed lack of a cusp (Buchholz et al. 2009; Do et al. 2009; Bartko et al. 2010). But a pre-existing core with radius smaller than the SMBH influence radius would have shrunk appreciably over a time of 10 Gyr due to gravitational encounters (Merritt 2010). We investigate the effect of such evolution on our NSC model by continuing the $N$-body integrations after the final infall event, for a time that corresponds to roughly 10 Gyr after scaling to the Milky Way. The core radius decreases by roughly a factor of two in this time, bringing it to a size that is more consistent with the observed core size. The density profile beyond the core remains nearly unchanged.

Since Galactic globular clusters are almost exclusively ancient objects with typical ages $10-13$ Gyr (e.g., Rosenberg et al. 1999), the merger model predicts that the bulk of the nuclear population is in old stars. Accordingly, using Hubble Space Telescope Near-Infrared Camera and Multiobject spectrometer (NICMOS) imaging of the inner $30 \mathrm{pc}$ of the Galaxy, we show that the luminosity function of the Milky Way NSC is consistent with the hypothesis that a large fraction of its mass is in ancient stars.

The paper is organized as follows. The details of our initial models are given in Section 2. Section 3 describes our simulations and results. Section 4 is devoted to the study of the collisional evolution of the NSC following its formation. The implications of our results in the contest of NSCs and Galactic center dynamics are discussed in Section 5. Section 6 sums up.

\section{INITIAL CONDITIONS}

In the following, we describe direct N-body simulations that were used to study the consecutive infall and merging of a set of 12 globular clusters each starting from a galactocentric distance of $20 \mathrm{pc}$. The total mass of the 12 , tidally-truncated clusters sums to $\sim 1.5 \times 10^{7} M_{\odot}$, roughly the observed mass of the Milky Way nuclear star cluster (Schödel et al. 2009). We chose 12 because this 
number permits a particularly elegant algorithm for generating the orbital initial conditions that does not favor any particular direction, as described in Section 2.2. After the first cluster had spiraled in to the center and reached a nearly steady state (as evaluated via Lagrange radii), we added the second cluster. This procedure was iterated until 12 clusters accumulated and merged in the inner region of the galaxy where we initially placed a central SMBH. Snapshots from the simulations are given in Figure 1. This scheme has been adopted to represent a realistic frame where the time interval between two consecutive globular cluster infalls and mergers to the center is longer than the time required for a single globular cluster to reach a quasi-steady state following its merger into the growing NSC. In any case, the results of the following simulations are robust and not depend (as far as the structure of the emerging NSC is concerned) much on specific initial conditions, as we discuss at the end of Subsection 3.1

We now outline the details of the initial conditions adopted in the simulations.

\subsection{The Galactic Model}

The nuclear bulge is distinguished from the larger Galactic bulge (effective radius $\sim 1 \mathrm{kpc}$ ) by its flat disklike morphology, high stellar densities, and a history of continuous star formation. The nuclear bulge dominates the inner $300 \mathrm{pc}$ of the Milky Way and it appears as a, distinct, massive disk-like complex of stars and molecular clouds which is, on a large scale, symmetric with respect to the Galactic center. It consists of an $r^{-2} \mathrm{nu}-$ clear stellar cluster within the inner $\sim 30 \mathrm{pc}$, a larger nuclear stellar disk and a nuclear molecular disk of same size (radius $\sim 200 \mathrm{pc}$ and scale height $\sim 45 \mathrm{pc}$ ). The total stellar mass and luminosity of the nuclear bulge are $1.5 \times 10^{9} \mathrm{M}_{\odot}$ and $\sim 2.5 \times 10^{9} \mathrm{~L}_{\odot}$, respectively (Launhardt et al. 2002). The $r^{-2}$ density distribution holds only within the NSC in the central $\sim 30 \mathrm{pc}$, while, at larger radii, the mass distribution is dominated by the nuclear stellar disk which has essentially a flat density profile (Schödel et al. 2011). The initial conditions for the galaxy in our simulations model the nuclear stellar disk and they omit the central NSC. Accordingly, they correspond to a shallow density cusp around a SMBH, which is included as a massive particle, $M_{\bullet}=4 \times 10^{6} \mathrm{M}_{\odot}$, located at the origin.

We adopted a truncated power-law model for this component:

$$
\rho_{g x}(r)=\tilde{\rho}\left(\frac{r}{\tilde{r}}\right)^{-\gamma} \operatorname{sech}\left(\frac{r}{r_{\text {cut }}}\right) .
$$

where $\tilde{\rho}=400 \mathrm{M}_{\odot} / \mathrm{pc}^{3}$ is the density at $r=\tilde{r}=$ $10 \mathrm{pc}$, and the truncation function is the same used by McMillan \& Dehnen (2005). Since $\operatorname{sech}(x) \approx 1-\frac{x^{2}}{2}$ for $x \ll 1$, the model is essentially a power law at $r \ll r_{\text {cut }}$, but it tends exponentially to zero for $r \gg r_{\text {cut }}$. We chose $\gamma=0.5$, corresponding to the shallowest power law consistent with an isotropic velocity distribution in a point-mass potential. The resulting model implies a mass density at $10 \mathrm{pc}$ similar to what is found in the Galaxy outside the NSC $\left(\sim 400 M_{\odot} / \mathrm{pc}^{3}\right)$. We chose $r_{\text {cut }}=22 \mathrm{pc}$ which gives a total mass of the (truncated) galactic model equal to $9.1 \times 10^{7} \mathrm{M}_{\odot}$.
In order to generate a Monte-Carlo realization of the distribution function corresponding to the truncated density profile of equation (44) we followed the method described in Szell et al. (2005). Using Eddington's formula, it can be shown that the cumulative fraction of stars at radius $r$ with velocities less than $v$ is:

$$
\begin{aligned}
& F(<v, r)=1-\frac{1}{\rho} \int_{0}^{E} d \phi^{\prime} \frac{d \rho}{d \phi^{\prime}} \times \\
&\left\{1+\frac{2}{\pi}\left[\frac{v / \sqrt{2}}{\sqrt{\phi^{\prime}-E}}-\tan ^{-1}\left(\frac{v / \sqrt{2}}{\sqrt{\phi^{\prime}-E}}\right)\right]\right\},
\end{aligned}
$$

where $E=\frac{1}{2} v^{2}+\phi(r)$ and $\phi(r)$ is the total gravitational potential produced by the stars and the SMBH. Once the positions are assigned, equation (5) can be numerically solved to distribute the particles in velocity space.

The number of particles used to represent the galaxy was $N=240,000$, which implies a mass of $\sim 380 \mathrm{M}_{\odot}$ for each particle in the system.

\subsection{The Globular Cluster Model}

The globular clusters were initially placed on circular orbits with orbital radii $r_{0}=20 \mathrm{pc}$. In order not to favor any particular direction for the inspiral, the orbital angular momenta were selected in the following way (e.g., Gualandris \& Merritt 2009). The surface of a sphere can be tessellated by means of 12 regular pentagons, the centers of which form a regular dodecahedron inscribed in the sphere. The coordinates of the centers of these pentagons were identified with the tips of the 12 orbital angular momentum vectors. In this way, the inclination and longitude of ascending node of each initial orbit were determined. The choice of circular orbits was motivated by the well-known effect of orbital circularization due to dynamical friction (Casertano et al. 1987; Ibata \& Lewis 1998; Hashimoto et al. 2003).

At a distance of $20 \mathrm{pc}$ from the Galactic center, a globular cluster would already have been subject to tidal forces from the galaxy and the SMBH, and its total mass and radius would be less than their original values when the globular cluster was far from the center. We assumed that the central properties of the globular clusters were unaffected by tidal forces during the inspiral to $20 \mathrm{pc}$, and adopted values characteristic of massive clusters: central velocity dispersion $\sigma_{K}=35 \mathrm{~km} \mathrm{~s}^{-1}$ and core radius $r_{K}=0.5 \mathrm{pc}$. If the dimensionless central (King) potential is $W_{0}=8$, the total mass works out to be $m \approx 4 \times 10^{6} \mathrm{M}_{\odot}$. This value of $\sigma_{K}$ is roughly two times the maximum value of $\sim 18 \mathrm{~km} \mathrm{~s}^{-1}$ listed in Harris (2010)'s compilation of Galactic globular clusters properties, while the core radius is roughly equal to the median value in that compilation. Our choice of such a large value for $\sigma_{K}$ is justified by the fact that only massive clusters, if they are compact enough, could have arrived in the central regions of the Galaxy in a reasonable time without being destroyed by Galactic tidal forces in the process (Miocchi et al. 2006 and $\S$ 5.1).

We then needed to generate equilibrium models for globular clusters with these same central properties, but with total masses and limiting (tidal) radii consistent with the known tidal forces from the Galaxy model at 20 pc. This is not a completely straightforward exercise, since the gravitational force from the globular cluster act- 


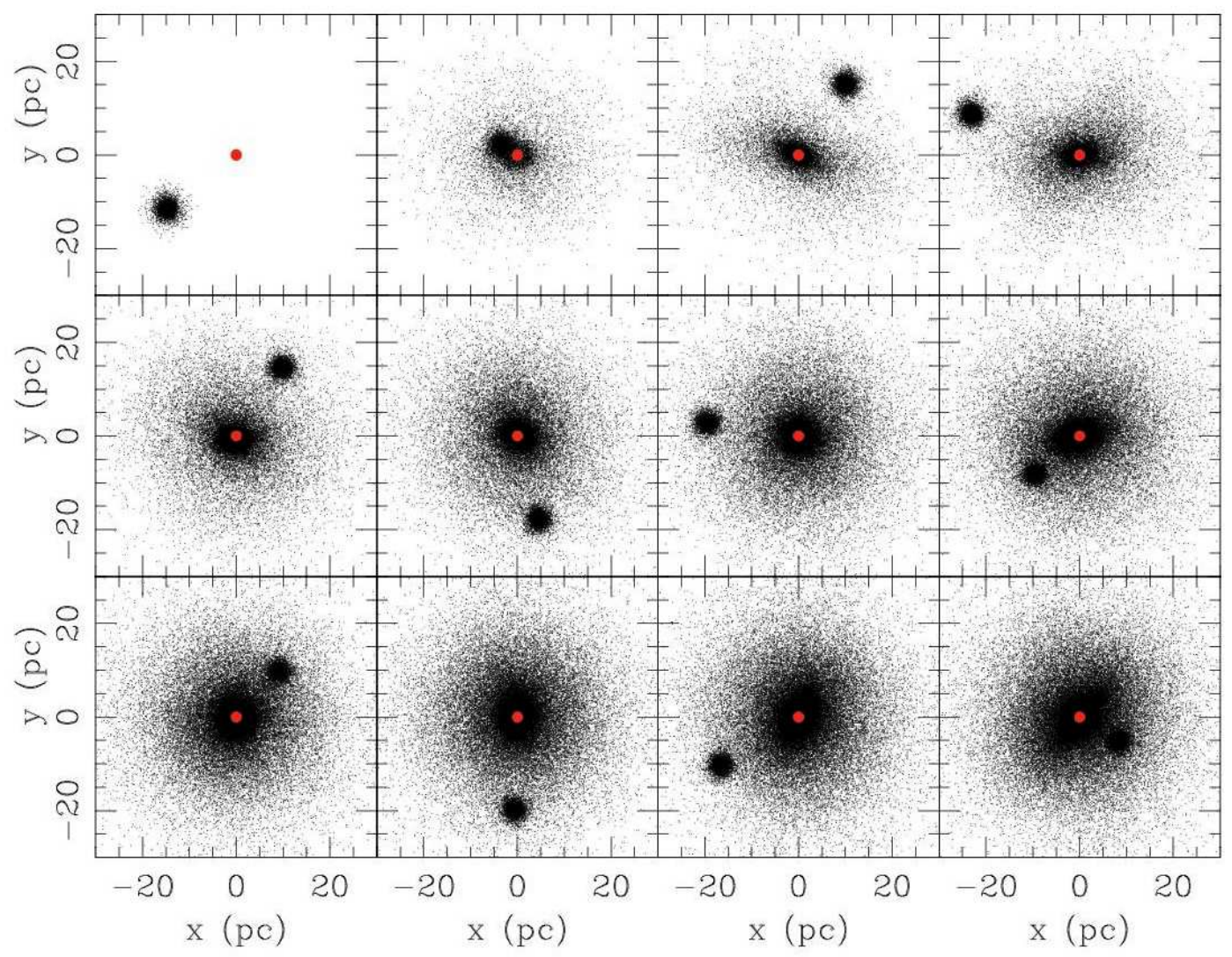

FIG. 1. - Snapshots from the $N$-body integrations, projected onto a fixed $(x-y)$ plane at the start of each infall event. Only particles coming originally from the infalling clusters are displayed. The SMBH is shown as the red circle.

ing on a star at the cluster's limiting radius, $r_{T}$, depends both on $r_{T}$ and on the cluster mass $m_{T}$ within $r_{T}$, and $m_{T}$ is a function of $r_{T}$.

We proceeded in the following way. We first assumed $r_{T} \gg r_{K}$. In this case, a King-like model satisfies the following relation between $m_{T}$ and $r_{T}$ :

$$
G m_{T} \approx \frac{1}{2} \sigma_{K}^{2} r_{T} .
$$

Given this relation, the tidal radius can then be related to the Galactic potential $\phi$ and density $\rho$ by (e.g., King 1962)

$$
r_{T}=\frac{1}{\sqrt{2}} \sigma_{K}\left[\frac{3}{r_{0}}\left(\frac{d \phi}{d r}\right)-4 \pi G \rho\right]^{-1 / 2} .
$$

Using the galaxy mass distribution of equation (4) and considering the presence of the SMBH, but ignoring the truncation function, we find

$$
\frac{d \phi}{d r}=\frac{8 \pi}{5} G \tilde{\rho} \tilde{r}\left(\frac{r}{\tilde{r}}\right)^{\frac{1}{2}}+\frac{G M_{\bullet}}{r^{2}},
$$

giving a limiting radius of

$$
r_{T}=\frac{1}{\sqrt{2}} \sigma_{K}\left[\frac{4 \pi}{5} G \tilde{\rho}\left(\frac{r_{0}}{\tilde{r}}\right)^{-1 / 2}+\frac{3 G M_{\bullet}}{r_{0}^{3}}\right]^{-1 / 2}
$$

and a tidally-truncated mass from equation (6). Adopting a distance $r_{0}=20 \mathrm{pc}$ we find $r_{T} \approx 8 \mathrm{pc}$ and
$m_{T} \approx 1.1 \times 10^{6} \mathrm{M}_{\odot}$; in other words, roughly $3 / 4$ of the globular cluster mass would have been removed in the process of inspiralling to $20 \mathrm{pc}$.

We then equated this $m_{T}$ with the mass of a new King model having the same core properties:

$$
m_{T}=m_{K} \equiv \rho(0) r_{K}^{3} \mu\left(W_{0}\right) \approx \frac{9}{4 \pi G} \sigma_{K}^{2} r_{K} \mu\left(W_{0}\right) .
$$

Here $\mu\left(W_{0}\right)$ is a function of the dimensionless central potential that is tabulated by King (1966). Since all the quantities in equation (10) are known except for $W_{0}$, we can solve for this variable, and find $W_{0}=5.8$. The three parameters $\left(W_{0}, r_{K}, \sigma_{K}\right)$ then uniquely define the King model that was used to generate the initial conditions of the globular clusters.

As previously stated, the mass of the single particle in the galaxy was $380 \mathrm{M}_{\odot}$. For the particles in the clusters we choose $200 \mathrm{M}_{\odot}$, approximately one half of that value. With this choice, the total number of particles in each globular cluster was 5,715 with 740 particles contained within the cluster core. 

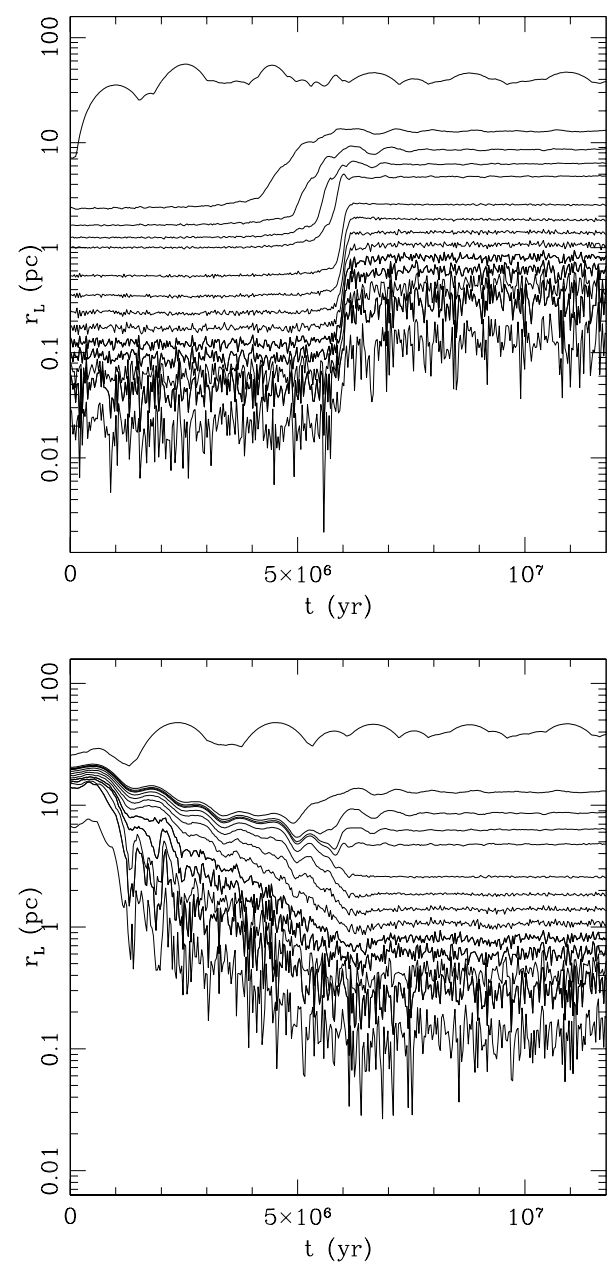

FIG. 2.- Lagrange radii of stars from the first cluster to arrive at the center of the galaxy. In the upper panel the radii are computed with respect to the center of density of the globular cluster, while in the lower panel they are computed with respect to the SMBH. The time for each cluster to reach a steady state after its disruption is a few Myr.

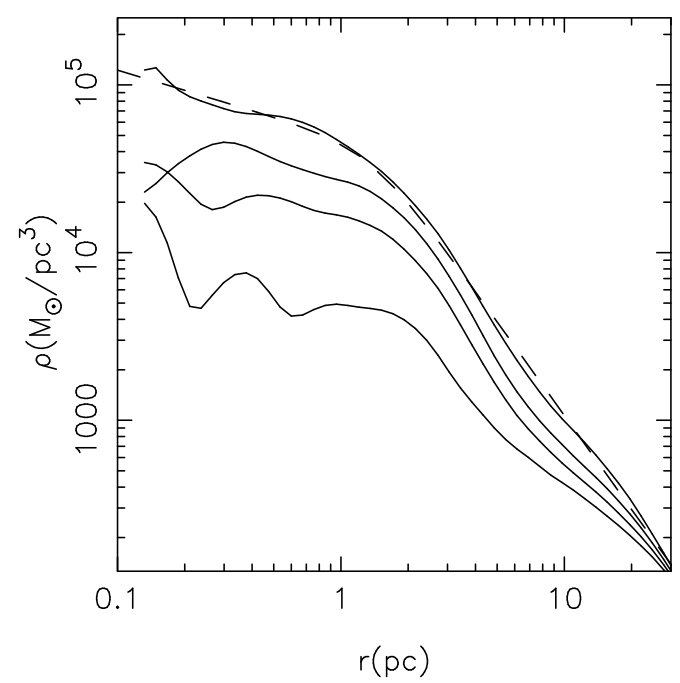

FIG. 3.- Density profile of the NSC after 3, 6, 9 and 12 infall events. The central density grows with time. The dashed line is the fit to the NSC density profile obtained at the end of the entire simulation using the broken power-law model of equation (11).

\section{3. $N$-BODY SIMULATIONS}

Our simulations were performed by using $\phi$ GRAPE (Harfst et al. 2007), a direct-summation code optimized for computer clusters incorporating the GRAPE specialpurpose accelerators (Makino \& Taiji 1998). The code implements a fourth-order Hermite integrator with a predictor-corrector scheme and hierarchical time stepping. The accuracy and performance of the code are set by the time-step parameter $\eta$ and the smoothing length $\epsilon$. In what follows, we set $\eta=0.01$ and $\epsilon=0.02 r_{K}\left(10^{-2} \mathrm{pc}\right.$ in our case), With these choices, energy conservation was typically $\lesssim 0.01 \%$ during each merging event. The simulations were carried out using the 32-node GRAPE cluster at the Rochester Institute of Technology, and also on computers containing Tesla C2050 graphics processing units at Sapienza-Universitá di Roma. In the latter integrations, $\phi$ GRAPE was used with SAPPORO, a CUDA library that emulates double-precision force calculations on single precision hardware (Gaburov et al. 2009).

During each infall event, we followed the evolution of the system until the globular cluster had reached the center of the galaxy and established an approximately steady state. This condition was verified by studying the time evolution of the globular cluster Lagrange radii, constructed both with respect to the center of density of the cluster (as defined by the algorithm in Casertano \& Hut 1985), and with respect to the central SMBH. When the Lagrange radii had reached nearly constant values, the next globular cluster was introduced. Figure 2 plots the time evolution of Lagrange radii for the first infall event. The figure shows that each merging episode lasts approximately $10^{7} \mathrm{yr}$ and that the time scale for a globular cluster to reach a steady state following its disruption is indeed very short, of the order of Myr.

We evaluated the $N$-dependence of our results by simulating the first three infalls using the same orbital initial conditions but with ten times more particles to represent the clusters. Comparing the density profile of the NSC after the three infalls with that obtained in the original integrations did not reveal any significant differences between the two cases.

\subsection{Results: density profiles}

Figure 3 shows the density profile of the system after the complete merging of $3,6,9$ and 12 clusters. We fitted the spatial density of the final system, within $10 \mathrm{pc}$ around the SMBH, using the broken power law model (e.g., Saha 1992; Zhao 1996):

$$
\rho(r)=\rho_{b}\left(\frac{r}{r_{b}}\right)^{-\gamma_{i}}\left[1+\left(\frac{r}{r_{b}}\right)^{\alpha}\right]^{\left(\gamma_{i}-\beta\right) / \alpha},
$$

where $\gamma_{i}$ is the slope of the inner density profile, $\beta$ the external slope and $\alpha$ is a parameter that defines the transition strength between inner and outer power laws. The best-fit parameters were $\rho_{b}=4.1 \times 10^{4} \mathrm{M}_{\odot} / \mathrm{pc}^{3}$, $r_{b}=1.5 \mathrm{pc}, \gamma_{i}=0.45, \beta=1.90$ and $\alpha=3.73$. The model corresponding to this set of parameters is given by the dashed line in Figure 3 .

Figure 4 (upper panel) plots the spatial density at the end of the simulation over a wider radial range than in Figure 3. We fitted the total density as a superposition of two parametric models, one intended to represent the 

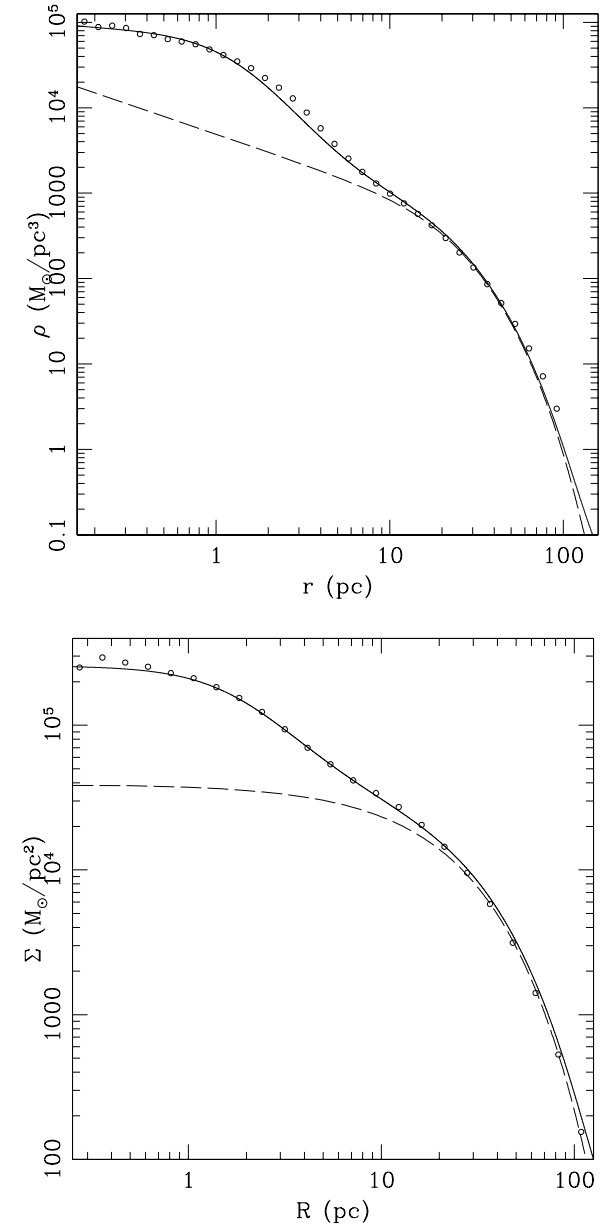

FIG. 4.- Spatial (upper panel) and projected (lower panel) density profiles at the end of the simulation. In each panel, the empty circles give the density profile of the $N$-body model, the solid lines give the best fitting model to the entire system (galaxy+NSC) and the dashed curves give the fit to the density profile of the galaxy, see text for explanation.

NSC and the other the galaxy. For the NSC we adopted the modified Hubble law (Rood et al. 1972):

$$
\rho_{c l}(r)=\rho_{0, c l}\left[1+\left(\frac{r}{r_{0, c l}}\right)^{2}\right]^{-\frac{3}{2}},
$$

with best fitting parameters $\rho_{0, c l}=7.46 \times 10^{4} \mathrm{M}_{\odot} / \mathrm{pc}^{3}$ and $r_{0, c l}=1.4 \mathrm{pc}$. The galaxy remained well-fit by the initial "truncated" power law of equation (1), when $\tilde{\rho}=$ $9.91 \times 10^{2} \mathrm{M}_{\odot} / \mathrm{pc}^{3}, \tilde{r}=10 \mathrm{pc}, \gamma=0.69$, and $r_{\text {cut }}=$ $16.3 \mathrm{pc}$.

The lower panel of Figure 4 shows the projected density of the $N$-body model at the end of the simulation. We again fit this profile with a two-component model. For the NSC we used

$$
\Sigma_{c l}(R)=\Sigma_{0, c l}\left[1+\left(\frac{R}{R_{0, c l}}\right)^{2}\right]^{-\zeta},
$$

while the projected density profile of the galaxy was rep-
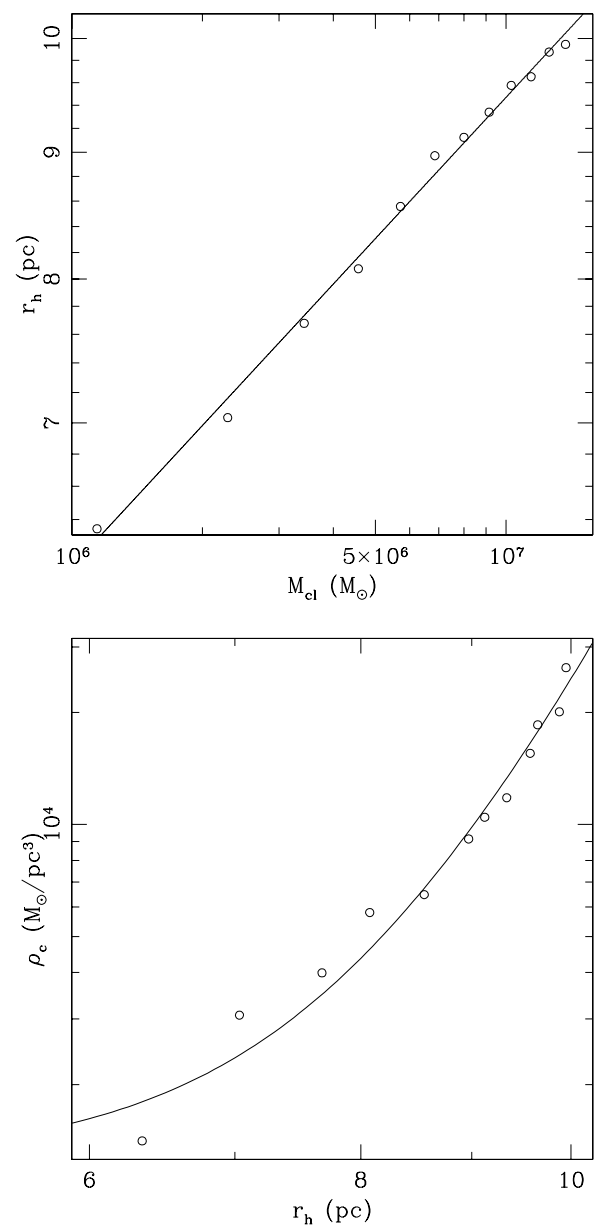

FIG. 5.- Upper panel: empty circles represent the half mass radius of the central NSC as a function of the total mass of the same system. The solid line represents the scaling relation given in equation (16). Lower panel: the core density of the cluster versus its half mass radius (empty circles). The solid line shows the $\rho_{c}-r_{h}$ relation given by equation (17).

resented by a Sérsic law:

$$
\Sigma_{g x}(R)=\Sigma_{0, g x} \exp \left[-b\left(\frac{R}{R_{0, g x}}\right)^{\frac{1}{n}}+b\right],
$$

with

$$
b=2 n-\frac{1}{3}+\frac{0.009876}{n} .
$$

The best-fit parameters were $\Sigma_{0, c l}=2.18 \times 10^{5} \mathrm{M}_{\odot} / \mathrm{pc}^{2}$, $R_{0, c l}=1.99 \mathrm{pc}$ and $\zeta=1.03$ for the NSC; $\Sigma_{0, g x}=$ $7.31 \times 10^{3} \mathrm{M}_{\odot} / \mathrm{pc}^{2}, b=1.68, R_{0, g x}=32.3 \mathrm{pc}$ and $n=1.003$ for the bulge.

Remarkably, our simulations result in a final density profile having nearly the same power-law index beyond $\sim$ $0.5 \mathrm{pc}$ as observed $\left(\Sigma(r) \sim r^{-1}\right.$; Becklin \& Neugebauer 1968; Haller et al. 1996). In addition, the central region $\left(r<r_{b}\right)$ of our model exhibits a shallow density profile (or core) near the SMBH, also in agreement with observations (Buchholz et al. 2009). The core radius in our model ( $\sim 2 \mathrm{pc})$ is somewhat larger than the observed core, of radius $\sim 0.5$ pc. In $\$$ we show that two-body relaxation would cause such a core to shrink over Gyr time scales, as the density evolves toward, but does not 

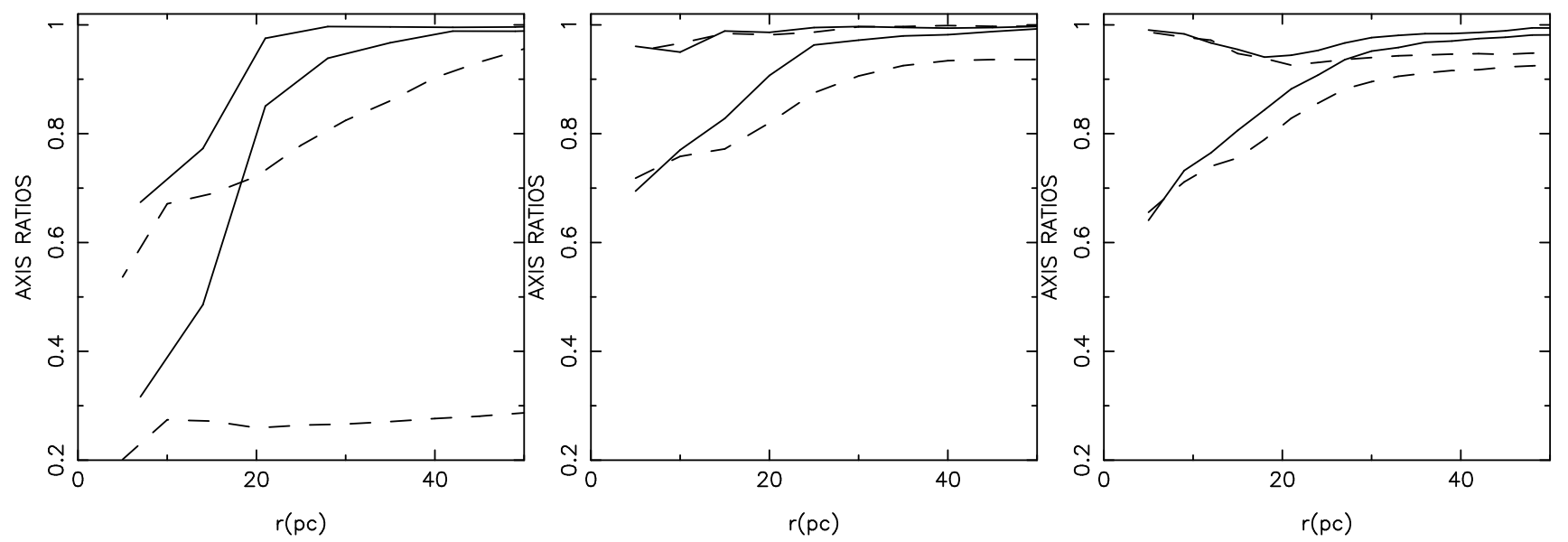

Fig. 6.- Axial ratios of the $N$-body system as a function of galactocentric radius computed after 1 (left panel), 6 (middle panel) and 12 (right panel) infalls. Solid curves correspond to the entire model (i.e., galaxy plus the NSC), while dashed curves gives the axis ratios of NSC only. After the first infall the NSC is strongly triaxial in the inner regions, but appears nearly oblate at the end of the simulation.

fully reach, a collisional steady state.

In the upper panel of Figure 5 the half-mass radius $\left(r_{h}\right)$ of the NSC component is plotted as a function of the NSC mass $\left(M_{c l}\right)$ at the end of each infall. At any time, the NSC mass is given by the sum of the accumulated globular cluster masses. A good fit to the data is obtained by

$$
r_{h}=0.45\left(\frac{M_{c l}}{M_{\odot}}\right)^{0.19} \mathrm{pc},
$$

represented by the solid line. The dependence of $r_{h}$ on $M_{c l}$ is weak, due to the fact that the size of the NSC is determined essentially by the fixed tidal field from the $\mathrm{SMBH}$.

Assuming for the growing NSC the density law of equation (12), the core density can be defined as $\rho_{c}=\rho_{0, c l} / 2^{\frac{3}{2}}$ and the values of $\rho_{c}$, obtained after the end of each infall, can be plotted as a function of the half mass radius of the same system (bottom panel of Figure 5). These data are well fit by

$$
\rho_{c}=\left[1.2 \times 10^{3}+1.1 \exp \left(\frac{r_{h}}{1 \mathrm{pc}}\right)\right] \frac{\mathrm{M}_{\odot}}{\mathrm{pc}^{3}},
$$

shown as solid curve in the figure.

We also carried out a separate simulation in which all 12 clusters were placed at the same time on their initial orbits. Such initial conditions are hardly realistic; we mention the outcome briefly here since it supports the robustness of the results obtained from the more realistic initial conditions. The "contemporaneous" infall model produced a very similar final density profile, with a core of radius $\sim 1$ pc and a $\rho \sim r^{-2}$ falloff at larger radii.

\subsection{Results: morphology of the NSC}

Observationally constraining the morphology and kinematics of galactic nuclei is a fundamental step toward understanding their origin. Unfortunately, as a consequence of the strong interstellar extinction in the plane of the Milky Way, our knowledge of the size and morphology of the Galactic NSC are limited. Kinematic modeling of proper-motion data derived from the dominant population of old, mostly giant stars reveals a nearly spherical system of low central concentration exhibiting slow, approximately solid-body rotation, of amplitude $\sim 1.4 \mathrm{~km} \mathrm{~s}^{-1} / \operatorname{arcsec}$ (Trippe et al. 2008; Schödel et al. 2009).

Aspherical NSCs are commonly observed in external galaxies. In a sample of 9 edge-on nucleated late-type galaxies, Seth et al. (2006) reported that three of these galaxies (IC 5052, NGC 4206, and NGC 4244) have NSCs with significantly flattened isophotes and evidence for multiple structural components. In addition, one of these galaxies (NGC 4206) showed possible indication of AGN activity, suggesting the presence of a SMBH within the core of the central cluster. The NSC of the face-on galaxy M33, in which a SMBH is not detected (Merritt et al. 2001; Gebhardt et al. 2001), is also known to be elongated along an axis parallel to the major axis of the galaxy (Lauer et al. 1998; Matthews 1999).

We quantified the model shape in our simulation by constructing isodensity contours and also by the moment-of-inertia tensor (e.g., Katz 1991; Poon \& Merritt 2004; Antonini et al. 2009), as described in what follows: the symmetry axes are calculated as

$$
\tau_{1}=\sqrt{I_{11} / I_{\max }}, \tau_{2}=\sqrt{I_{22} / I_{\max }}, \tau_{3}=\sqrt{I_{33} / I_{\max }},
$$

where $I_{i i}$ are the principal moments of the inertia tensor and $I_{\max }=\max \left\{I_{11}, I_{22}, I_{33}\right\}$; particles are then enclosed within the ellipsoid $x^{2} / \tau_{1}{ }^{2}+y^{2} / \tau_{2}{ }^{2}+z^{2} / \tau_{3}{ }^{2}=r^{2}$. These previous two steps were iterated until the values of the axial ratios had a percentage change of less than $10^{-3}$. Finally, we define $a>b>c$ letting $c / a=\min \left\{\tau_{1}, \tau_{2}, \tau_{3}\right\}$ and $b / a$ the intermediate value. We also define the triaxiality via the parameter $T \equiv\left(a^{2}-b^{2}\right) /\left(a^{2}-c^{2}\right)$. Oblate and prolate galaxies have $T=0$ and 1 , respectively. The value $T=0.5$ corresponds to the 'maximally triaxiality' case.

The results are summarized in Figure 6 which displays the axial ratios of the NSC as a function of radius and at different times. The model morphology evolves from an initially strong triaxiality (after the first infall) into a more quasi-axisymmetric, oblate shape. In particular, the morphological structure of the final product (right panel) is very similar to that after the 6th infall event 
(middle panel). This shows that the NSC is transformed into a nearly oblate system $(T \lesssim 0.2$ at $r<20 \mathrm{pc})$ after few infalls $(\sim 4)$, but its shape remains essentially unchanged from that point on. In the outer regions ( $\gtrsim 20 \mathrm{pc}$ ), the system remained instead nearly spherical for the entire course of the simulation.

\subsection{Results: kinematics}

Figure 7 illustrates the kinematics of the final model. The upper-left panel of the figure shows the onedimensional velocity dispersions along, and perpendicular to, the radius vector, defined with respect to the SMBH. For $r<0.3 \mathrm{pc}$ the system is quite isotropic, but it becomes tangentially anisotropic for $0.3 \mathrm{pc}<r<20 \mathrm{pc}$. At larger radii the system is again roughly isotropic. The upper right panel of Fig. 7] shows the amplitude and orientation of the major axes of the $2 \mathrm{~d}$ velocity ellipsoid, derived from the $(x, y)$ velocities. The length of the plotted axes is proportional to the velocity dispersion. The tangential anisotropy is apparent.

The lower panels of Figure 7 show the anisotropy parameter

$$
\beta=1-\frac{\sigma_{t}^{2}}{2 \sigma_{r}^{2}},
$$

as a function of distance from the $\mathrm{SMBH}$, and as a function of time at two different distances, $10 \mathrm{pc}$ and $20 \mathrm{pc}$. In the radial range $0.3-40 \mathrm{pc}, \beta$ is negative and the system is tangentially anisotropic. The anisotropy grows with the number of infall events.

\section{COLLISIONAL EVOLUTION OF THE NUCLEAR STAR CLUSTER}

The simulations of globular cluster inspiral described above took place in a short enough span of time that two-body relaxation effects could be ignored. The local relaxation time can be defined as (Spitzer 1987)

$$
t_{\mathrm{r}}=\frac{0.33 \sigma^{3}}{G^{2} \rho m_{\star} \ln \Lambda}
$$

where $m_{\star}$ is the stellar mass, $\rho$ is the mass density and $\sigma$ is the one-dimensional velocity dispersion. Near the influence radius of a $\mathrm{SMBH}$, the Coulomb logarithm can be approximated as $\ln \Lambda=\ln \left(r_{\text {inff }} \sigma^{2} / 2 G m_{*}\right)$. The relaxation time at the influence radius of $\mathrm{Sgr} \mathrm{A}^{*}, r_{\mathrm{infl}}=$ $2-3 \mathrm{pc}$, is $t_{\mathrm{r}, \text { inf }} \sim 20-30 \mathrm{Gyr}$, assuming a stellar mass of $1 M_{\odot}$ (Merritt 2010). This is roughly $2 \times 10^{5}$ times the period of a circular orbit at $r_{\text {infl }}$. In our $N$-body simulations, the relaxation time is shorter (compared with the crossing time) by a factor of approximately 200, the mass of a single cluster particle in solar masses; in other words, it is roughly $10^{3}$ times the crossing time at $r_{\text {infl }}$.

In the absence of large-scale changes to the gravitational potential, an $N$-body model like ours continues to evolve due to gravitational encounters. The evolution that occurs should mimic the evolution that would take place in the real system, of much larger $N$, if the unit of time is taken to be the relaxation time (e.g., Aarseth \& Heggie 1998).

In the Milky Way, the relaxation time is short enough that significant evolution of the stellar distribution near the SMBH would take place over the age of the galaxy. The distribution of late-type stars in the Milky Way
NSC exhibits a nearly flat core of radius $\sim 0.5 \mathrm{pc}$ (Buchholz et al. 2009). In a time of $10 \mathrm{Gyr}$, such a core would shrink, as the stellar density evolved toward the Bahcall-Wolf (1976) $\rho \sim r^{-7 / 4}$ form inside $\sim 0.2 r_{\text {infl }}$. Since the density profile beyond the core is observed to have roughly this slope (Oh et al. 2009), such evolution would tend to preserve the outer slope while gradually reducing the size of the core. A core of initial radius $1-2$ $\mathrm{pc}$ is expected to reach a size of $\sim 0.5 \mathrm{pc}$, the size of the observed core, after $\sim 10$ Gyr (Merritt 2010). These arguments motivated us to continue the integration of our $N$-body models after the final inspiral event. Figure 8 shows the density profile of the NSC at different times during its post-merger evolution. At the end of this inte-

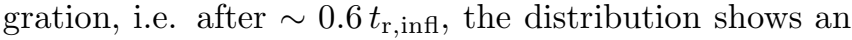
inner core of size $\sim 0.2 \mathrm{pc}$, substantially reduced from its initial value of $\sim 1.5 \mathrm{pc}$. The bottom panel of Figure 8 plots the evolution of the break radius, $r_{b}$, of the best fitting broken power law profile as a function of time. The value of the break radius can be used as an approximate estimate of the model core radius. The time dependence of the core radius is well described by an exponential:

$$
r_{b}(t)=1.57 \mathrm{pc} \exp \left[-t /\left(0.25 t_{\mathrm{r}, \mathrm{inf}}\right)\right] .
$$

As expected, the slope of the density profile outside the core remains nearly unchanged during this evolution, $\rho \simeq$ $r^{-1.8}$.

A core radius of $\sim 0.5 \mathrm{pc}$ is reached after a time of $\sim 0.25 t_{\mathrm{r}, \text { infl. }}$ Scaled to the Milky Way, this time would be $5-8$ Gyr.

Of course, in the real galaxy, it is likely that cluster inspiral would occur more or less continuously over the lifetime of the galaxy. Our separation of the evolution into an inspiral phase, followed by a relaxation phase, is artificial in this sense. Nevertheless it is reasonable to draw the conclusion that the size of the core resulting from the combined effects of cluster inspiral and relaxation would be somewhat smaller than the $\sim 1.5 \mathrm{pc}$ that we found above, and therefore, closer to the observed core size of $\sim 0.5 \mathrm{pc}$.

Figure 9 shows the morphological evolution of the NSC during the relaxation phase: the radial dependence of the axis ratios (upper panel) and the triaxiality parameter (lower panel). There is essentially no evolution in the intermediate axis ratio. However, in the innermost regions of the model, the shortest axis length significantly increases with time. Two-body relaxation results in an evolution toward quasi-spherical symmetry, but at the end of the simulation the model has not yet reached this final state, still exhibiting some non-negligible triaxiality. The final model is nearly oblate with $0.3 \lesssim T \lesssim 0.1$. We note that such deviations from spherical symmetry, although mild, might be large enough to substantially enhance the rates of stellar capture and disruption by the $\mathrm{SMBH}$ with respect to the same rate computed in collisionally resupplied loss cone theories (Merritt 2012).

Figure [10 illustrates the evolution of the velocity anisotropy profile, $\beta(r)$, during the post-merger phase. Relaxation tends to drive the velocity distribution toward isotropy, causing $\beta$ to increase toward zero (this dynamical trend is similar to that described

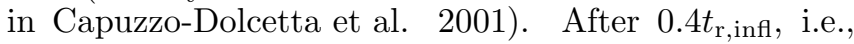
$\sim 10$ Gyr, there remains only a small bias toward tangen- 
tial motions, $\beta \sim-0.1, r \lesssim 10$ pc. The final anisotropy profile is consistent with measurements of the Galactic center (Schödel et al. 2009; Merritt 2010). In the radial range $1^{\prime \prime}-10^{\prime \prime}$, the late-type stars are observed to have a mean projected anisotropy of $1-\left\langle\sigma_{T}^{2} / \sigma_{R}^{2}\right\rangle=-0.124_{-1.05}^{0.098}$ with $\sigma_{R}$ and $\sigma_{T}$ the radial and tangential velocity dispersions in the plane of the sky.

\section{DISCUSSION}

\subsection{Cluster inspiral times}

For the model described here to be viable, globular clusters must spiral into the central regions of their parent galaxy in a time shorter than $\sim 10^{10} \mathrm{yr}$. A rough estimate of the time for a globular cluster of mass $m$ at initial radius $r_{0}$ to inspiral into the center of a galaxy as a result of dynamical friction is (Merritt et al. 2004):

$$
\Delta t=\frac{C(\gamma)}{\ln \Lambda} \frac{r_{a}^{3}}{m}\left(\frac{\rho}{G}\right)^{1 / 2}\left(\frac{r_{0}}{r_{a}}\right)^{(6-\gamma) / 2}
$$

This expression assumes circular orbits, a total mass density of the galaxy that satisfies $\rho(r)=\rho_{a}\left(r / r_{a}\right)^{-\gamma}$, and a frictional force that is due entirely to stars with velocities less than the orbital velocity of the globular cluster, and Coulomb logarithm $\ln \Lambda=6.5$ Antonini \& Merritt 2011). The coefficient $C$ in equation (5.1) is a weak function of $\gamma$, with $C=(3.9,3.6,4.2)$ for $\gamma=(1,1.5,2)$. Writing $\tilde{r}_{a} \equiv r_{a} /(1 \mathrm{kpc}), \quad \tilde{\rho}_{a} \equiv \rho_{a} /\left(1 M_{\odot} p c^{-3}\right), \tilde{m} \equiv$ $m / 10^{6} M_{\odot}$ and $\tilde{r_{0}} \equiv r_{0} / r_{a}$, the time for a globular cluster to spiral in is:

$$
\Delta t \approx 9 \times 10^{9} \mathrm{yr} \quad \tilde{r}_{a}^{3} \tilde{\rho}_{a}^{1 / 2} \tilde{m}^{-1} \tilde{r}_{0}^{(6-\gamma) / 2} .
$$

Although the initial distribution of globular clusters is not known, a reasonable assumption is that it follows the distribution of the total baryonic mass predicted by the standard cosmological model.

Let $\Delta t_{h}$ be the time for clusters initially within $r_{h}$, the half mass radius of the galactic bulge, to spiral to the center. Within $\Delta t_{h}$, the forming nucleus has a luminosity comparable to the luminosities of the surviving clusters. An estimate of $r_{h}$ is $r_{e}$, the observed projected half-light (effective) radius; the stellar density at $r=r_{e}$ is $\tilde{\rho}_{e} \approx$ $14.1 \tilde{r}_{e}^{-2.519}$, with $r_{e}$ the half light radius in $\mathrm{kpc}$ and $\tilde{\rho}_{e}$ the stellar density in units of solar masses per cubic parsec. Luminosity profiles for $r<r_{e}$ are well approximated as power laws with $1<\gamma<2$ in the same galaxies (Terzíc \& Graham 2005). Thus,

$$
\Delta t \approx 3 \times 10^{10} \mathrm{yr} \quad \tilde{r}_{e}^{1.75} \tilde{m}^{-1} .
$$

For globular clusters of mass $m=10^{5}\left(10^{6}\right) M_{\odot}, \Delta t<$ $10^{10}$ yr requires $r_{e}<140(520)$ pc. For comparison bulges of $\mathrm{S} 0-\mathrm{Sb}$ galaxies and early type galaxies with $-20<M_{B}<-16$ have $200 \lesssim r_{e} \lesssim 5000$ pc , albeit with a large scatter (Faber et al. 1997; Ferrarese et al. 2006; Graham \& Worley 2008). Though crude, this calculation implies that a significant fraction of the globular clusters in faint galaxies and bulges would have spiraled to the center in $10^{10} \mathrm{yr}$.

The dynamical evolution of the Galactic globular cluster system has been already investigated by many authors using different schemes and approximations (e.g., Murali \& Weinberg 1997;
Takahashi \& Portegies Zwart 2000; Shin et al. 2008). None of these previous calculations were however designed to track the cluster orbits down to the sphere of influence of the central black hole. A more accurate estimate of orbital inspiral times for clusters in the Galactic bulge is obtained in what follows by adopting a model for the background distribution that reproduces more accurately the observed stellar density profile in the inner $\sim 1 \mathrm{kpc}$ of the Milky Way.

We numerically integrated the equations of motion of a cluster in a fixed potential including the contribution of dynamical friction:

$$
\begin{aligned}
\ddot{\mathbf{r}} & =-\nabla \phi+\mathbf{a}_{\mathrm{df}}, \\
\mathbf{a}_{\mathrm{df}} & =-4 \pi G^{2} m \rho(r) F(<v, r) \ln \Lambda \frac{\mathbf{v}}{v^{3}} .
\end{aligned}
$$

Here $\rho(r)$ is the mass density of background stars, $F(<$ $v, r)$ is the fraction of stars with local velocities less than that of the cluster and $\ln \Lambda$ is the Coulomb logarithm. For the background distribution we adopted the density model:

$$
\begin{aligned}
\rho(r)= & \rho_{\mathrm{NSD}}+\rho_{\mathrm{GB}}=400 \frac{M_{\odot}}{\mathrm{pc}^{3}}\left(\frac{r}{10 \mathrm{pc}}\right)^{-0.5} \exp \left[-\frac{r}{70 \mathrm{pc}}\right] \\
& +6 \frac{M_{\odot}}{\mathrm{pc}^{3}} \exp \left[-\left(\frac{r}{800 \mathrm{pc}}\right)^{2}\right],
\end{aligned}
$$

where $\rho_{\text {NSD }}$ is the density of the nuclear stellar disk and the second term, $\rho_{\mathrm{GB}}$, represents the contribution of the Galactic bulge which becomes significant for $r \gtrsim 200 \mathrm{pc}$. This density profile approximates the mass model shown in Figure 14 of Launhardt et al. (2002). We chose $m=4 \times 10^{6} M_{\odot}$ for the untruncated mass of the globular cluster. In order to include the effect of tidal truncation, at any time the corresponding tidally-truncated mass $\left(m_{T}\right)$, defined in equation (6), was computed and assigned to the infalling globular cluster (i.e., $m=m_{T}$ ) if $m_{T}<m$.

Figure 11 plots the orbital evolution of globular clusters with different values of the central velocity dispersion and starting on circular orbits of radius $1 \mathrm{kpc}$. Globular clusters with central velocity dispersion larger than about $15 \mathrm{kms}^{-1}$ would reach the center in less than a Hubble time if they are initially inside the Galactic bulge.

We stress that the calculations presented in Figure 11 give a conservative upper limit to the decay times for various reasons. Some of these are: i) the presence of gas in the central regions could dramatically decrease the sinking time of a stellar cluster (Ostriker 1999); ii) the background distribution is assumed to be spherical, but in a more realistic triaxial model the decay time could be greatly reduced (Pesce et al. 1992).

In addition, the sinking times given in Figure 11 are a clear overestimate of the real dynamical friction decay times since they were evaluated considering the Chandrasekhar local approximation for tidally truncated globular clusters on circular orbits (orbits that minimize the dynamical friction effect for a given orbital energy). More accurate orbit integrations, apt to follow the entire evolution of an orbit through the singularity, show that globular clusters on radial orbits decay in a time more than 30 times faster than on circular orbits of same energy (Arca-Sedda \& Capuzzo-Dolcetta 2011). All this 

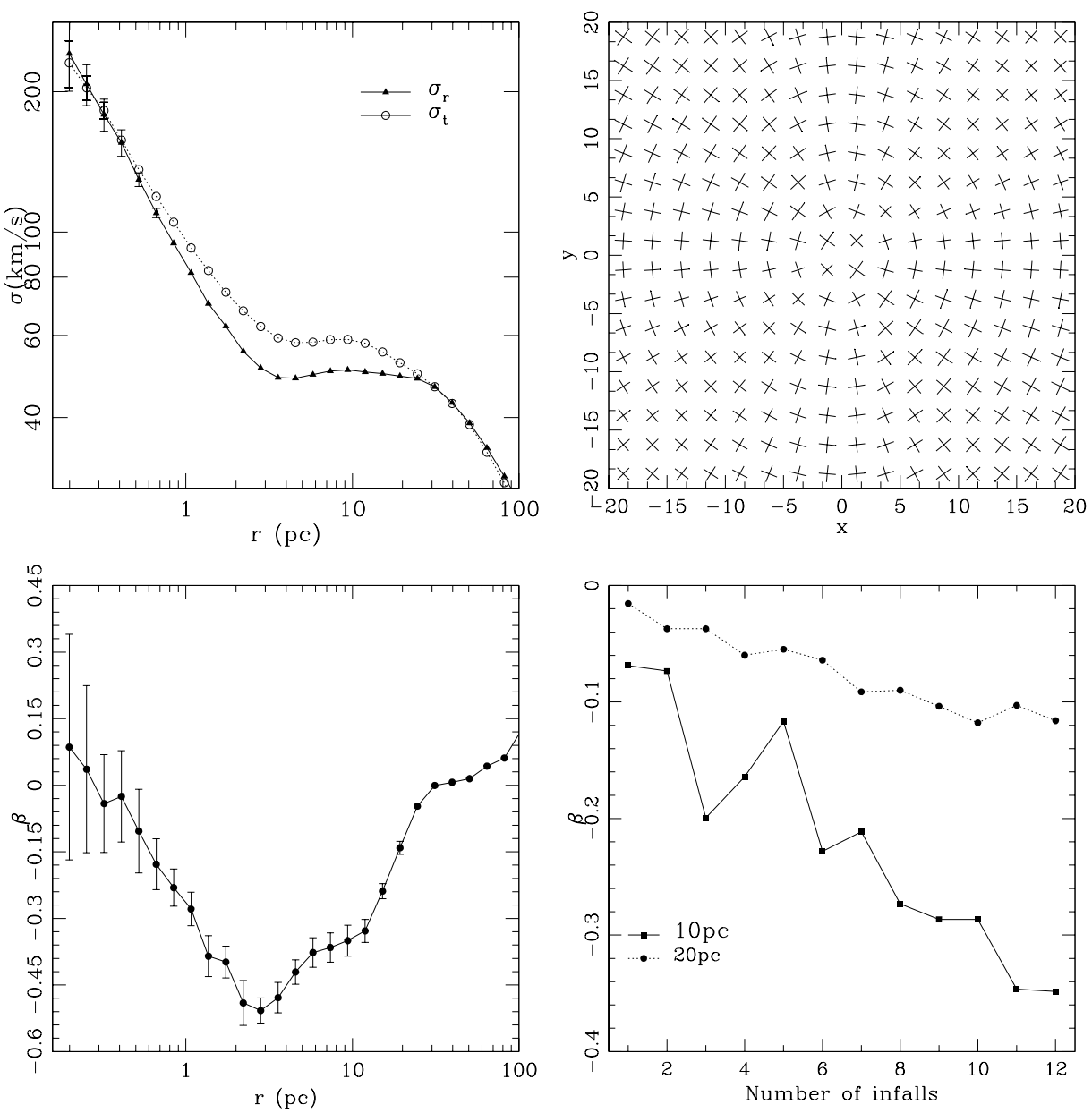

FIG. 7.- Upper left: radial $\left(\sigma_{r}\right)$ and tangential $\left(\sigma_{t}\right)$ velocity dispersions plotted as functions of the distance from the SMBH. Upper right: map of the principal axes of the $2 \mathrm{D}$ velocity ellipses derived from the $(x, y)$ velocity components. Lower left: Anisotropy parameter $\beta$. Lower right: The anisotropy parameter evaluated at 10 and $20 \mathrm{pc}$ versus time. All plots were derived form the model at the end of the 12 th inspiral event.

convince us that the decay toward the MW central region of sufficiently massive GCs has occurred on a time significantly shorter than the age of the Galaxy.

\subsection{Star Formation History of the Milky Way Nuclear Cluster}

The NSC at the center of the Milky Way appears to have undergone continuous star formation over the last 10 Gyr (e.g., Serabyn \& Morriss 1996). The evidence that a large fraction of the Galactic center mass was formed in situ at an approximately constant rate over the last 10 Gyr has been extensively used in the past to argue against the merger model for the Milky Way NSC (e.g., Milosavliević 2004; Navakshin et al. 2009) .

In this section, we compare the observed luminosity function (LF) of the stellar populations at the Galactic center with synthetic LFs obtained for different star formation scenarios. Our analysis suggests the possibility that about $1 / 2$ of the Milky Way nuclear population consists of $\sim 10$ Gyr old stars brought in by infalling globular clusters, while the remaining mass is due to continuous star formation.

Dereddened LFs of the observed populations were generated by Hubble Space Telescope NICMOS data taken from Figer et al. (2004). The fields are all within the central 30 pc of the Galactic center; their exact locations are given in Table 1 and shown in Figure 1 of that paper. Data are complete at the $50 \%$ level at $m_{\mathrm{F} 205 \mathrm{~W}}=19.3$, averaged over all fields. The details of the technique used to generate both model and observed LFs are also described in Figer et al. (2004) and are summarized in what follows.

The LFs of the Galactic center populations are constructed under the assumption that each star has the intrinsic colors of a red giant and by subtracting reddening values for each star corresponding to its color in $H-K$ or $m_{\mathrm{F} 160 \mathrm{~W}}-m_{\mathrm{F} 205 \mathrm{~W}}$.

Geneva isochrones are used to model the LFs corresponding to different star formation histories. The Geneva stellar evolutionary models are described in Shaller et al. (1992), Schaerer et al. (1993a,b), Charbonnel et al. (1993) and Mevnet et al. (1994). We adopted a mass spectrum of stars with a Salpeter (Salpeter 1955) power law index (i.e., $d N / d m=m^{-1.35}$ ) and upper and lower mass cutoff of $120 M_{\odot}$ and $0.1 M_{\odot}$ respectively. The Geneva models are used to convert these masses to the absolute magnitudes in the $V$ band that are subsequently transformed in the $K$ band through a lookup table that relates color index to mag- 

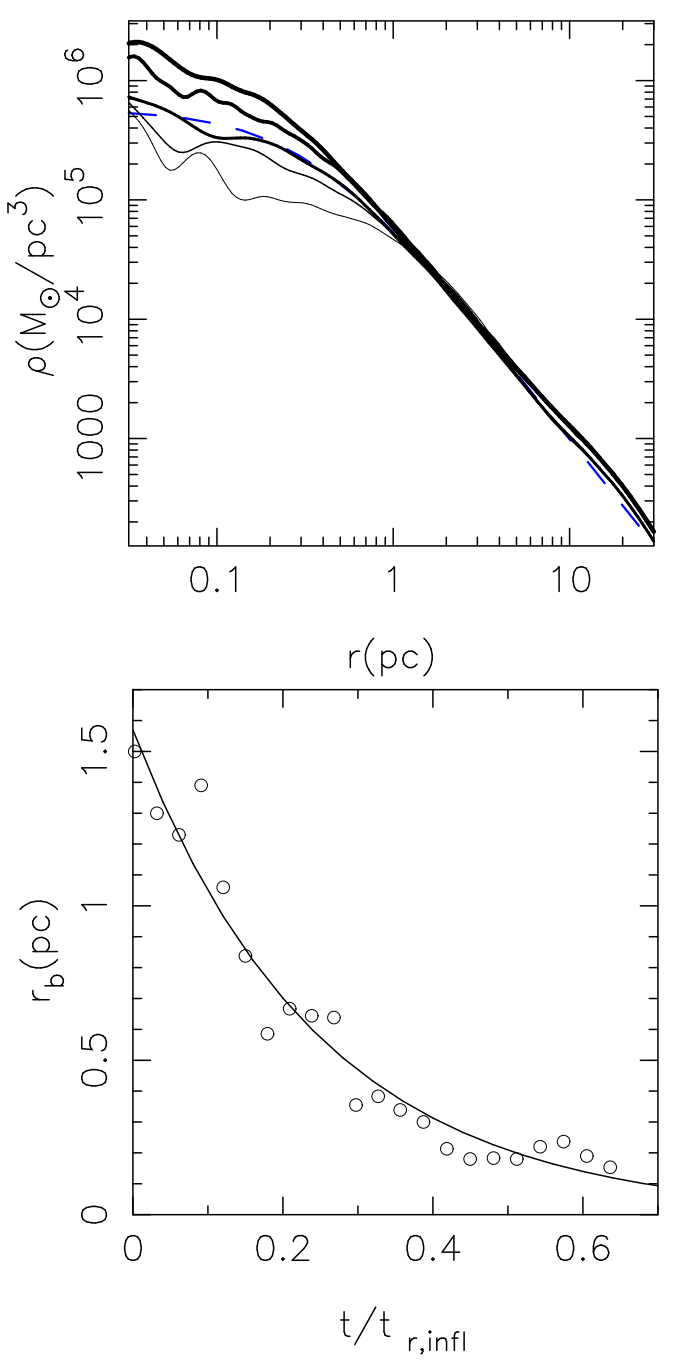

FIG. 8.- Post-infall evolution of the $N$-body model. Upper panel shows the density profile at four times: $t=$ $(0.12,0.24,0.36,0.48,0.6)$ in units of the relaxation time at the influence radius; line thickness increases with time. The dashed (blue) curve shows a fit of the density to the broken power-law model of equation (11) at $t \approx 0.36 t_{\mathrm{r}, \text { infl, }}$, i.e., $\sim 10 \mathrm{Gyr}$ when scaled to the Milky Way. Lower panel plots the break (core) radius as a function of time. The solid line is the best-fit exponential, equation (21).

nitude. We then sum over the histogram to produce the $\mathrm{LF}$ of each star formation event and sum the individual LFs to derive the LF for a given star formation scenario.

Figure 12 displays the results of this study for various star formation histories, with the model counts modified by the observed completeness fractions from Figer et al. (1999). From the top to the bottom panels, the plots correspond to star formation models in which: i) the entire mass is build up by ancient ( $\sim 10$ Gyr old) stars, possibly brought into the Galactic center by infalling globular clusters; ii) the mass model is composed by ancient globular clusters stars plus stars formed via continuous star formation; iii) some $(\sim 1 / 3)$ of the mass is formed during a starburst at $\sim 1$ Gyr (e.g., Siouwerman et al. 1999), while the remainder is due to continuous star formation and to an ancient burst at $10 \mathrm{Gyr}$; iv) the entire mass is formed through continuous star formation over the last 10 Gyr.
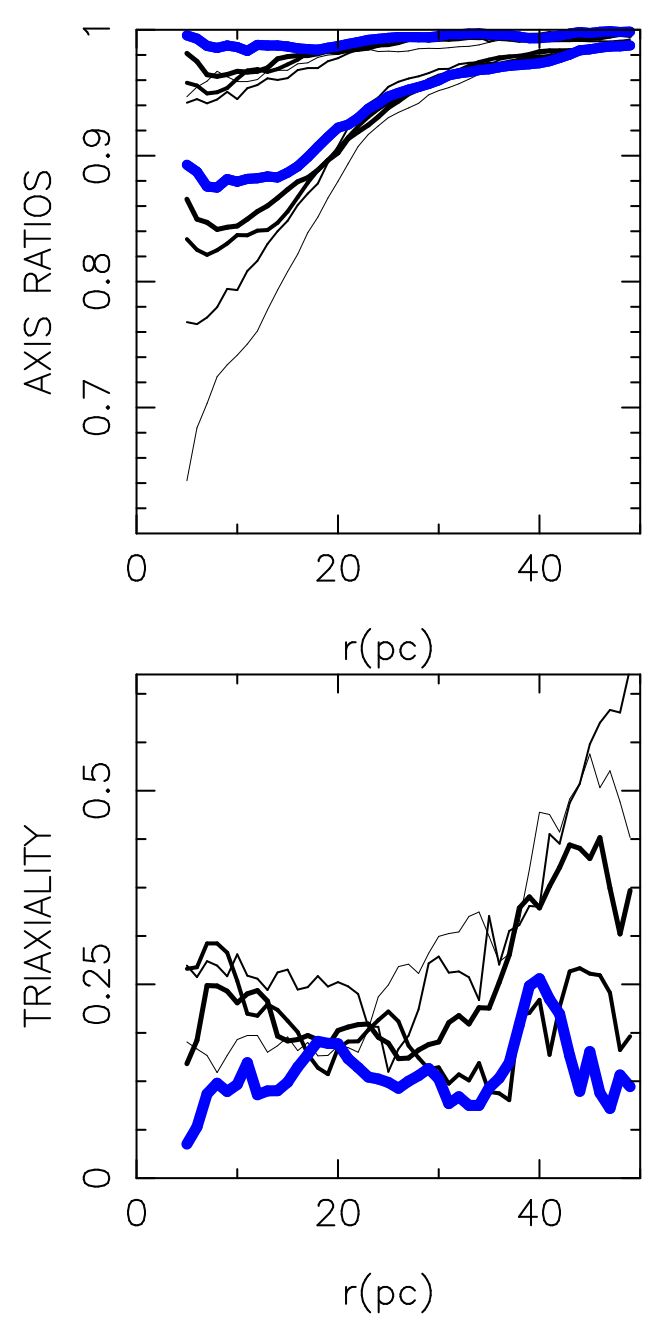

FIG. 9.- Evolution of the model axis ratios (upper panel) and triaxiality parameter (lower panel), as functions of radius, in the post-merger phase. Times are the same as in Figure 8 line thickness increases with time. Thickest blue lines correspond to the the final model.

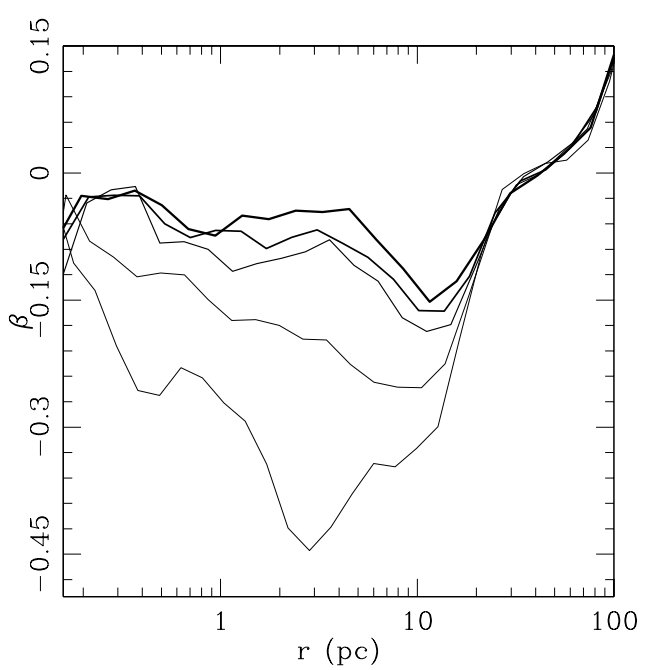

FIG. 10.- Evolution of the anisotropy parameter $\beta$ during the post-merger phase. Times shown are the same as in Figure 8 line thickness increases with time. 

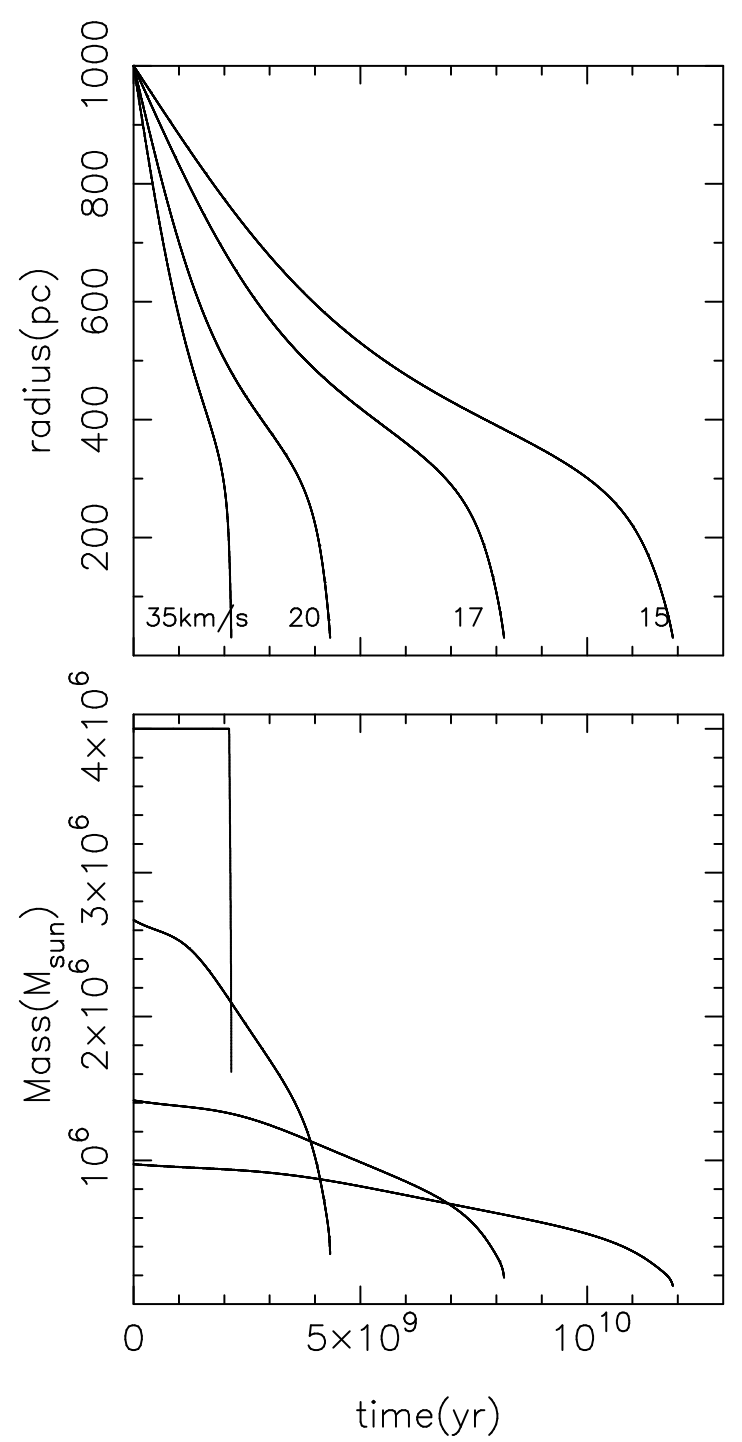

FIG. 11.- Evolution of orbital radius (upper panel) and mass (lower panel) for globular clusters with different central velocity dispersions starting on circular orbits of radius $1 \mathrm{kpc}$. These computations include the effect of tidal truncation due to the galactic potential (see text for explanation). Globular clusters with velocity dispersion larger than approximately $15 \mathrm{kms}^{-1}$ would spiral to the center within $10^{10} \mathrm{yr}$.

The counts at faint magnitudes $\left(K_{0}>15\right)$ are controlled by ancient star formation, while the counts at the bright end $\left(K_{0}<8\right)$ are controlled by the extent of recent star formation activity. The brightness of the red clump (at $K_{0} \sim 12$ ) is related instead to the extent of intermediate age star formation activity.

The figure shows that the ancient burst model, corresponding to a NSC composed of only ancient stars, fails at reproducing the observed LF. This model overestimates the counts at faint magnitudes and it does not reproduce the number of counts seen in the bright end. Our analysis rules out the possibility that the nuclear population consists entirely of ancient stars. On the other hand, star formation models in which ancient bursts are accompanied by continuous star formation at other times, produce a LF essentially indistinguishable from that obtained when the mass is entirely due to continuous star formation. All these latter models fit quite well number counts and shape of the observed LF. We conclude that the observed Galactic center LF is consistent with a star formation history in which a large fraction of the mass consists of ancient ( $\sim 10 \mathrm{Gyr})$ stars. A similar result was obtained recently by Pfuhl et al. (2011). These authors constructed a complete Hertzprung Russel diagram of the red giant population within $1 \mathrm{pc}$ from Sgr $\mathrm{A}^{*}$ and found that about $80 \%$ of the stellar mass in these regions was formed more than 5 Gyr ago.

\subsection{Mass-radius relation}

In Figure 13 the mean half-mass radius is plotted against total mass for nuclei (filled circles) globular clusters (open circles) and ultra compact dwarfs (UCDs, star symbols). We overplot the track followed by the NSC in our simulation during the infall events (purple-continue curve) and during relaxation (continue-blue line). The structural properties of the NSC formed in our simulations (blue-filled circle) are in good agreement with those of real NSCs.

From the figure we can see that the faintest nuclei have roughly the same mass as a typical globular cluster. The size distributions for the nuclei and globular clusters also overlap, although the clusters in the Galaxy have halfmass radii of $3 \mathrm{pc}$, irrespective of mass, while the nuclei follow a relation of the form $r_{h} \propto \sqrt{M}$. Fainter than a few million solar masses, the nuclei and globular clusters have comparable sizes (Hasegan et al. 2005).

We now consider the merger model for nucleus formation in the absence of a SMBH. In this case one can derive a simple recursive relation between the mass and radius of the NSC during its formation. The radius of the nucleus increases with increasing total mass, or light, as globular clusters merge. After the merger, its final energy, $E_{f}$, equals the energy of the nucleus before the merger, $E_{i}$, plus the energy brought in by the globular cluster. This energy has two components: the internal energy or binding energy $E_{b}$, and the orbital energy just before the merger, $E_{o}$. From conservation of energy:

$$
E_{f}=E_{i}+E_{o}+E_{b}
$$

Just before the merger, the orbital energy is $E_{o}=$ $\alpha G m M_{i} / 2 R_{i}$, where $M_{i}$ and $R_{i}$ are the mass and radius of the nucleus, respectively, $m$ is the mass of the globular cluster, and $\alpha$ is a constant of order unity (Hausman \& Ostriker 1978) that depends on the radius of the capture orbit - the radius at which the dominant influence on the trajectory of a globular cluster first comes from the nucleus. After the merger, the nucleus reaches a state of dynamical equilibrium quickly; the virial theorem implies $E_{f}=-G M_{f}^{2}$. The equations above permit expressing the mass, energy, and radius of the nucleus recursively as

$$
\begin{gathered}
M_{j+1}=(j+1) M_{1}, \\
j E_{j+1}=(j+\alpha) E_{j}+j E_{1}, \\
(j+1)^{2} R_{j+1}^{-1}=j(j+\alpha) R_{j}^{-1}+R_{1}^{-1}, j=1,2,3, \ldots
\end{gathered}
$$

where the subscript 1 denotes the initial nucleus, and, by assumption, $M_{1}=m$. At the time when a nucleus consists of few merged globular clusters, its mass and 

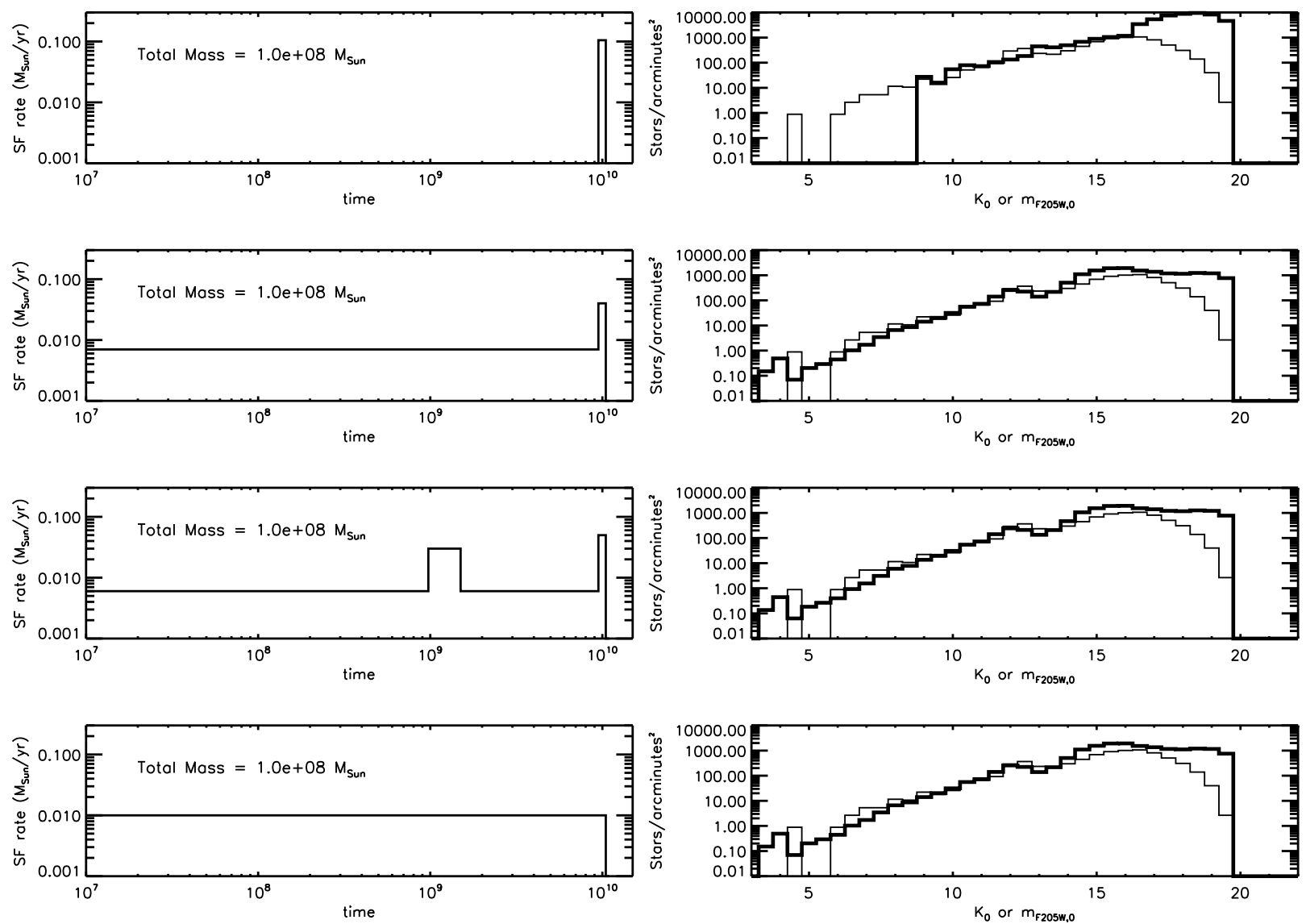

FIG. 12.- Left panels display different star formation scenarios in which, from top to bottom: the entire mass is build up by ancient ( $\sim 10$ Gyr old) stars, that we assume to be brought into the Galactic center by infalling globular clusters; half of the mass is contributed by old stars and half due to continuous star formation; at the mass contributed by continuous star formation and globular clusters we add stars formed during a starburst episode occurred at $\sim 1 \mathrm{Gyr}$; all the mass is contributed by continuous star formation. Right panels compare the observed LF of the Galactic center (light lines) with the LFs (heavy lines) resulting from the different star formation scenarios assuming Solar metallicity and canonical mass loss-rates in the Geneva models. Note that the data are much more than $50 \%$ incomplete for the faintest few bins. The models have not been scaled for mass, but rather have been rescaled along the vertical axis to mach the number counts in the $K=11.0$ bin. The star formation histories corresponding to the three bottom set of panels are essentially indistinguishable from each other and they all reproduce quite well the observed LF.

that of the next infalling globular cluster are comparable. In this case, equations (28130) imply $R \propto M^{0.5}$. However, after many mergers, $M \gg m$, and the relation steepens to $R \propto M$. For $\alpha=1.2$ and $5(25) 100$ mergers, equations (28,30) imply $R \approx 2(5) 10$ and $R \propto M^{p}$, $p=0.5(0.6) 0.7$. The typical half-mass radius of a globular cluster is about 3 pc (Jordán et al. 2005) so for a nucleus assembled from 25 mergers, $R \sim 15$ pc. This is in reasonable agreement with the measured sizes for the brighter nuclei. For $\alpha=1.2$, the expected scaling between $r_{h}$ and mass is shown by the blue dot-dashed curves in Figure 13. We show the predicted behavior for two assumptions for the mass, $m$, of the clusters which merge to form the nucleus: $10^{5}$ and $10^{6} M_{\odot}$. At least for $m=10^{6} M_{\odot}$, the agreement with the $r_{h}$-mass relation for real nuclei is remarkably good.

\section{SUMMARY}

We have used large-scale direct $N$-body simulations to test the merger model for the formation of the Milky Way nuclear star cluster (NSC). Our initial conditions consisted of a massive black hole (SMBH) at the center of a nearly homogeneous $N$-body system representing the nuclear stellar disk. Globular clusters were then added to this system, starting from circular orbits of radius 20 pc. The clusters were tidally limited by the external field to have a mass of $\sim 1.1 \times 10^{6} \mathrm{M}_{\odot}$ at the start. Infall was driven by dynamical friction, due to the stellar disk, and later also to the accumulated mass from the previouslymerged clusters. The clusters were fully disrupted by the SMBH at a radius of approximately one parsec. After 12 inspiral events, the accumulated mass of the NSC was about $1.5 \times 10^{7} M_{\odot}$, comparable with the actual mass.

The principle results of our study are summarized below.

1 The stellar system resulting from the consecutive mergers has a density that falls off as $\sim r^{-2}$, and a core of radius $\sim 1 \mathrm{pc}$. These properties are similar to those observed in the Milky Way NSC.

2 The morphology of the NSC evolved during the course of the infalls, from an early, strongly triaxial shape toward a more oblate/axisymmetric shape near the end of the merger process. Kinematically, the final system is characterized by a mild tangen- 


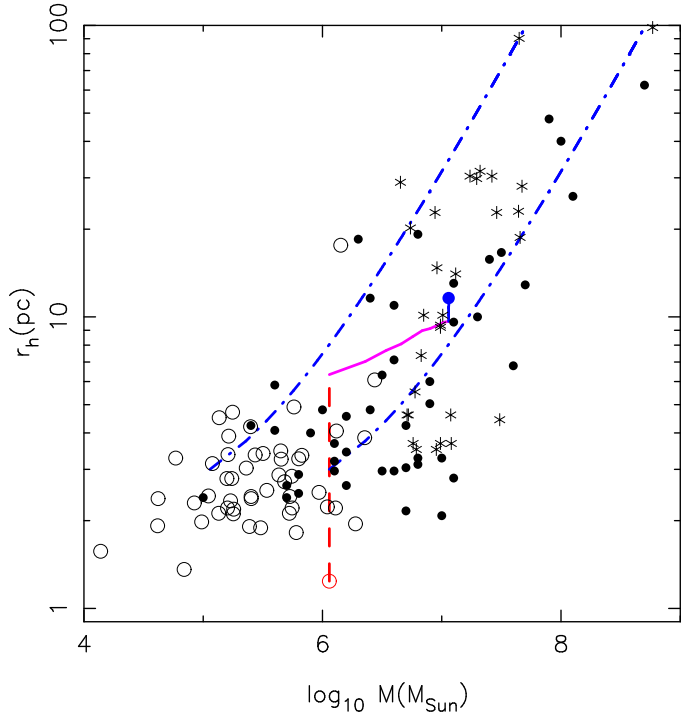

FIG. 13. - The measured mean half-mass radius (or effective radius) plotted against total mass for nuclei (filled circles), globular clusters (empty circles) and UCDs (stars symbols). Data points are from Forbes et al. (2008) and Côté et al. (2006). Blue dot-dashed curves show the predicted scaling in the merger model without SMBH for two different choices for the mass of merged clusters (see text for details). The red-open circle represents the initial globular cluster model in the $N$-body simulation. Purple and continue-blue curves are the evolutionary track of the NSC during its formation and during relaxation respectively. The filled blue circle represents the final product of our simulation.

tial anisotropy within the inner $30 \mathrm{pc}$ and a low degree of rotation.

3 In order to investigate the effect of gravitational encounters on the evolution of the NSC, we continued the $N$-body integrations after the final inspiral was complete. The core that had been created by the SMBH was observed to shrink by roughly a factor of two in 10 Gyr as the stellar density evolved toward a Bahcall-Wolf cusp. This final core size is essentially identical to the size of the core observed at the center of the Milky Way. The density profile outside the core remained nearly unchanged during this evolution. Gravitational encounters also caused the NSC to evolve toward spherical symmetry in configuration and velocity space.

4 Since Galactic globular clusters are almost exclusively ancient objects with ages $\sim 10-13$ Gyr, in the merger model a large fraction of the NSC mass is expected to be in old stars. We confronted this prediction with the observed luminosity function (LF) of the Milky Way NSC. Using stellar population models, we showed that the observed LF is consistent with a star formation history in which a large fraction (about $1 / 2$ ) of the mass consists of old $(\sim 10 \mathrm{Gyr})$ stars and the remainder from continuous star formation.

This research was supported by the National Science Foundation under grants AST 08-07910, 08-21141 and by the National Aeronautics and Space Administration under grant NNX-07AH15G. We are grateful to Don Figer that provided us with data and code used to generate Figure 12. We thank H. Perets, C. Trombley, E. Vasiliev for useful discussions and the referee, S. Portegies Zwart, for comments that helped to improve the paper.

\section{REFERENCES}

Aarseth, S. J. \& Heggie, D. C., 1998, MNRAS, 297, 794

Agarwal, M. \& Milosavljević 2011, ApJ, 729, 35

Antonini, F., Capuzzo-Dolcetta, R., \& Merritt, D., 2009, MNRAS, 399, 671

Antonini \& Merritt 2012, ApJ, 745, 83

Arca-Sedda, M. \& Capuzzo-Dolcetta, R., 2011, in preparation

Bahcall, J. N., \& Wolf, R. A. 1976, ApJ, 209, 214

Bartko et al. 2010, ApJ, 708, 834

Becklin, E. E., \& Neugebauer, G. 1968, ApJ, 151, 145

Bekki, K., Couch, W. J., Drinkwater, M. J., \& Shioya, Y. 2004 ApJ, 610, L13

Bekki, K. 2007, PASA, 24, 77B

Böker, Laine, S., van der Marel, R. P., Sarzi, M., Rix, H., Ho, L. C., \& Shields, J. C. 2002, AJ, 123, 1389

Böker, T. 2010, ihea.book, 99

Buchholz, R. M., Schödel, R., \& Eckart, A. 2009, A\&A, 499, 483

Capuzzo-Dolcetta, R., 1993, ApJ, 415, 616

Capuzzo-Dolcetta, R., Miocchi, P., 2008, MNRAS, 388, L69

Capuzzo-Dolcetta, R., Pucello, N., Rosato, V., \& Saraceni, F. 2001, Journal of Computational Physics, 174, 208

Carollo, C. M., Stiavelli, \& Mack, J. 1998, AJ, 116, 68

Casertano, S., Hut, P., 1985, ApJ, 298, 80

Casertano, S., Phinney, E. S., \& Villumsen, J. V., 1987, IAU

Symp. 127, Structure and Dynamics of Elliptical Galaxies, ed. T. de Zeeuw (Dordrecht: Reidel), 475

Charbonnel, C., Meynet, G., Maeder, A., Schaller, G., \& Schaerer, D. 1993, A\&AS, 101, 415

Côté et al. 2006 ApJS, 165, 57

Do, T., Ghez, A. M., Morris, M. R., Lu, J. R., Matthews, K., Yelda, S., \& Larkin, J. 2009, ApJ, 703, 1323

Emsellem, E. \& van de Ven, G. 2008, ApJ, 674, 653

Ebisuzaki et al. 2001, ApJ, 562, L19

Faber, S. M. et al. 1997, AJ, 114, 1771

Ferrarese, L. et al. 2006, ApJ, 644, L21

Figer, D. F., Kim, S. S., Morris, M., Serabyn, E., Rich, R. M., \& McLean, I. S. 1999, ApJ, 525, 750
Figer, D. F., Rich, R. M., Kim, S. S., Morris, M., \& Serabyn, E. 2004, ApJ, 601, 319

Forbes, D. A., Lasky, P., Graham, A. W., \& Spitler, L. 2008, MNRAS, 389, 1924

Gaburov, E., Harfst, S., \& Portegies Zwart, S. 2009, NewAstron. 14630

Gebhardt, K., Lauer, T. R., Kormendy, J., et al. 2001, AJ, 122, 2469

Genzel, R. et al. 2003, ApJ, 594, 812

Ghez, A. M. et al., 2008, ApJ, 689, 1044

Gillessen, S., Eisenhauer, F., Trippe, S., Alexander, T., Genzel, R., Martins, F., Ott, T. 2009, ApJ, 692, 1075

Graham, A. W. \& Spitler, L. R. 2009, MNRAS, 397, 2148

Graham, A., \& Worley, C. C. 2008, MNRAS, 388, 1708

Gualandris, A., \& Merritt, D., 2009, ApJ, 705, 361

Haller, J. W., Rieke, M. J., Rieke, G. H., Tamblyn, P., Close, L. \& Melia, F. 1996, ApJ, 456, 194

Harfst, S., Gualandris, A., Merritt, D., Spurzem, R., Portegies Zwart, S., \& Berczik, P. 2007, NewAstron. 12, 357

Harris, W. E., 1996 (2010 edition), AJ, 112, 1487

Hartmann, H., Debattista, V. P., Seth, A., Cappellari, M., \& Quinn, T. R., 2011, arXiv:1103.5464

Hashimoto, Y., Funato, Yoko, \& Makino, J. 2003, ApJ, 582, 196

Hassegan et al. 2005, ApJ, 627, 203

Hausman, M. A. \& Ostriker, J. P 1978, ApJ, 224, 320

Ibata, R. A., \& Lewis, G. F. 1998, ApJ, 500, 575

Jordán, A. et al., 2005, ApJ, 634,1002

Katz, N., 1991, ApJ, 368, 325

King, I. R., 1962, AJ, 67, 471

King, I. R., 1966, AJ, 71, 64

Lauer, T. R., Faber, S. M., Ajhar, E. A., Grillmair, C. J., \& Scowen, P. A. 1998, AJ, 116, 2263

Launhardt, R., Zylka, R., \& Mezger, P. G. 2002, A\&A, 384, 112

Loose, H. H., Kruegel, E., \& Tutukov, A. 1982, A\&A, 105, 342

Lotz J. M., Miller B. W., Ferguson H. C., 2004, ApJ, 613, 262 
Makino, J., \& Taiji, M. 1998, Scientific Simulations with Special-Purpose Computers-the GRAPE Systems, by Junichiro Makino, Makoto Taiji, pp. 248. ISBN 0-471-96946-X. Wiley-VCH , April 1998.,

Matthews, L. D., et al. 1999, 118, 208

McMillan, P. J., Dehnen, W., 2005, MNRAS, 363, 1205

Merritt, D., 2010, ApJ, 718, 739

Merritt, D., 2012, Black Holes and the Dynamics of Galactic Nuclei (Princeton University Press)

Merritt, D., Piatek, S., Portegies Zwart, S., \& Hemsendorf, M. 2004, ApJ, 608, L25

Merritt, D., Ferrarese, L., \& Joseph, C. L. 2001, Science, 293, 1116

Meynet, G., Maeder, A., Schaller, G., Schaerer, D., \& Charbonnel, C. 1994, A\&AS, 103, 97

Milosavljević, M. 2004, ApJ, 605, L13

Miocchi, P., Capuzzo-Dolcetta, R., Di Matteo, P., \& Vicari, A., 2006, ApJ, 644, 940

Murali, C. \& Weinberg, M. 1997, MNRAS, 291, 717

Nayakshin, S., Wilkinson, M. I., King, A. 2009, MNRAS, 398L, 54

Oh, S., Kim, S. S., \& Figer, D. F. 2009, JKAS, 42, 17

Ostriker, E. C. 1999, ApJ, 513, 252

Pesce, E., Capuzzo-Dolcetta, R., \& Vietri, M. 1992, MNRAS, 256, 368

Pfuhl, O. et al. 2011, ApJ, 741, 108

Poon, M. Y., \& Merritt, D. 2004, ApJ, 606, 774

Portegies Zwart, S. F.; Baumgardt, H.; McMillan, S. L. W. Makino, J.; Hut, P, \& Ebisuzaki, T, 2006 ApJ, 641, 319

Rood, H. J., Page, T. L., Kintner, E. C., \& King, I. R. 1972, ApJ, 175, 627

Rosenberg, A., Saviane, I., Piotto, G., \& Aparicio, A. 1999 AJ, 118,2306

Saha, P. 1992, MNRAS, 254, 132
Salpeter, E. E. 1955, ApJ, 121, 161

Schaerer, D., Charbonnel, C., Meynet, G., Maeder, A., \& Schaller, G. 1993a, A\&AS, 102, 339

Schaerer, D., Meynet, G., Maeder, A., \& Schaller, G. 1993b, A\&AS, 98, 523

Schaller, G., Schaerer, D., Meynet, G., \& Maeder, A. 1992 A\&AS, 96, 269

Schinnerer, E. et al. 2006, ApJ, 649, 181

Schinnerer E., Böker T., Meier D. S., \& Calzetti D. 2008, ApJL, 684, L21

Schödel, R., Eckart, A.. Alexander, T., et al. 2007, A\&A, 469, 125

Schödel, R., Merritt, D., \& Eckart, A. 2008, Journal of Physics Conference Series, 131, 012044

Schödel, R., Merritt, D., \& Eckart 2009 A\&A, 502, 91

Schödel, R. 2011, ASPC, 439, 222

Serabyn, E., \& Morriss, M. 1996, Nature, 382, 602

Seth A. C., Dalcanton J. J., Hodge P. W., Debattista V. P., 2006, AJ, 132,2539

Seth, A. et al. 2008, ApJ, 678, 116

Shin, J., Kim, S. S., \& Takahashi, K. 2008, MNRAS, 386, L67

Shlosman I., \& Begelman M. C. 1989, ApJ, 341, 685

Sjouwerman, L. O., Habing, H. J., Lindqvist, M., van Langevelde, H. J., \& Winnberg, A. 1999, ASPC, 186, 379

Spitzer, L. 1987, Dynamical evolution of Globular Clusters

(Princeton: Princeton Univ. Press)

Szell, A., Merritt, D., \& Kevrekidis, I. G. 2005, Physical Review Letters, 95, 081102

Takahashi, K., Portegies Zwart, S. F. 2000, ApJ, 535, 759

Tremaine, S. D., Ostriker, J. P., \& Spitzer, L., Jr. 1975, ApJ, 196, 407

Trippe et al. 2008, A\&A, 492, 419

Wehner, E. H. \& Harris, W. E. 2006, ApJ, 644, L17

Zhao, H. 1996, MNRAS, 278, 488 